THE IDEAL BODY SHAPE OF AFRICAN AMERICAN COLLEGE WOMEN

A Dissertation presented to the Faculty of the Graduate School

University of Missouri

In Partial Fulfillment of the Requirements for the Degree

Doctor of Philosophy

By

Rashanta A. Bledman

Dr. Laurie Mintz, Dissertation Supervisor

May 2011 
The undersigned, appointed by the dean of the Graduate School, have examined the thesis or dissertation entitled

\section{THE IDEAL BODY SHAPE OF AFRICAN AMERICAN COLLEGE WOMEN}

presented by Rashanta A. Bledman,

a candidate for the degree of Doctor of Philosophy in Counseling Psychology

and hereby certify that, in their opinion, it is worthy of acceptance.

Laurie B. Mintz, Ph.D.

Department of Educational, School, and Counseling Psychology

Mary J. Heppner, Ph.D.

Department of Educational, School, and Counseling Psychology

Norman C. Gysbers, Ph.D.

Department of Educational, School, and Counseling Psychology

Cynthia M. Frisby, Ph.D.

School of Journalism 


\section{ACKNOWLEDGEMENTS}

As I reflect upon my journey of completing this dissertation, I am amazed by the number of people, both family and friends, that have cheered me on and encouraged me throughout the process.

First, and foremost, I must thank God. His grace, mercy and blessings have been a constant in my life and without Him, I would not have made it this far.

My family has made me who I am today and I am truly blessed and honored to be an Akka Bledman. Carlyeon and Mayleen Akka Bledman, my parents. You sacrificed so much to provide me a better life. You never let me believe that any dream was too big or any goal was unattainable. You are my twin pillars of support and I am so blessed to have you in my life. I am proud to call you Daddy and Mommy. I love you.

Samantha Bledman, my big sister. I have always looked up to you because your strength and steadfastness amaze me. Your faith and beautiful smile make you shine as a

glowing example for all to see. Thank you so much for guiding me and advising me in so many aspects of my life. I love you.

Shanaka Bledman, my big sister. You have always taken care of me. You never judged me and always encouraged me to be strong and persevere. You are so giving and supportive to me and everyone in your life. Thank you for always answering the phone and listening to my troubles. I love you.

Aspaltha Bledman, my big brother. No matter how I feel, you can always bring a smile to my face. I never worry when I am with you, because I know that you will protect me like no one else can. You are the coolest big brother and I love you. 
Alanna Bledman, my niece. You continue to be my favorite person in the world. Despite being my niece, you still manage to take care of me, tell me a joke, remind me to rest, and make me laugh. I love being silly with you and am amazed at the woman you are becoming. I cannot wait to see you make your mark on the world. I love you.

Airica Hartsfield, my best friend. You are more than a friend, you are my sister. You are a wonderful example of determination and dedication. Your pursuit of your life goals inspires and encourages me. Thank you for always being there for me. I love you.

Karen Traylor, my bestie. You are an awesome woman and friend. You have encouraged me to "get it together" and "keep on keeping on!" Thank you for praying for me and with me and being such a great person in my life. You are a sister to me and I love you.

David Good-Cross, my bestie. You were there for me, personally and professionally, when I felt discouraged and needed advice. You helped guide me through graduate school and constantly assured me that my feelings were "totally normal." I am so proud of your achievements and so appreciative of how much you have helped me. You are a brother to me and I love you.

Specifically to this dissertation process, I would like to recognize my doctoral committee. I admire and respect each of you so much. You have been firm in your confidence in me from the beginning of this process. Your patience, excitement and encouragement have kept me motivated and I appreciate you.

I would be remiss if I did not acknowledge the MU Black Studies Department, Nathan Stephens and the MU Black Culture Center, MU Legion of Black Collegians, Dorienna Alfred and the MU Student Health Center, and Kurt Debord and the Lincoln University campus for your support and willingness to put up flyers and encourage 
women to participate in my study. I literally would have no data if not for you. Thank you!

During the statistical analyses portion of this project, I relied heavily on two individuals: Xinting Zhao and Susan Kashubeck-West. Xinting, your humor, sarcasm, and frank nature helped me to enjoy analyzing my data. You are incredibly intelligent and were a huge help. Susan, you answered all of my emails and phone calls with such patience and clarity. You have been my statistics guru from beginning to end. I cannot express enough appreciation.

Laurie Mintz, my advisor. I can honestly say that my graduate school experience and dissertation writing would not have been the same were it not for you, my wonderful advisor, mentor, and "Jewish mother." You have encouraged me and helped me to grow personally and professionally and have genuinely cared about me as a person. I cannot fully express in words how much you have touched my life. Your confidence in me has often given me the strength to complete the task at hand. You are a wonderful and amazing woman who inspires me to be my best. Thank you so much, Laurie. You have helped me more than you know.

Lastly, to the countless others, who include my extended family, church families, and Columbia, Missouri friends, thank you for your encouragement and support. 


\section{TABLE OF CONTENTS}

$\begin{array}{ll}\text { ACKNOWLEDGEMENTS } & \text { ii }\end{array}$

LIST OF TABLES vii

ABSTRACT viii

CHAPTER ONE: INTRODUCTION 1

CHAPTER TWO: REVIEW OF THE LITERATURE 7

$\begin{array}{ll}\text { Possible Protective Factors } & 10\end{array}$

$\begin{array}{ll}\text { Impact of Media } & 12\end{array}$

Black Women's Satisfaction with Their Bodies 14

$\begin{array}{ll}\text { Black Women's Dissatisfaction with Their Bodies } & 17\end{array}$

Black Women's Satisfaction with Specific Body Areas 23

$\begin{array}{ll}\text { Purpose of Current Investigation } & 25\end{array}$

CHAPTER THREE: METHODOLOGY 26

$\begin{array}{ll}\text { Participants } & 26\end{array}$

$\begin{array}{ll}\text { Instruments } & 27\end{array}$

$\begin{array}{ll}\text { Procedure } & 34\end{array}$

$\begin{array}{ll}\text { Statistical Analyses } & 36\end{array}$

CHAPTER FOUR: RESULTS 42

$\begin{array}{ll}\text { Data Cleaning and Screening } & 42\end{array}$

Study Question One $\quad 43$

$\begin{array}{ll}\text { Study Question Two } & 45\end{array}$

$\begin{array}{ll}\text { Study Question Three } & 46\end{array}$ 
Study Question Four

Study Question Five $\quad 48$

$\begin{array}{ll}\text { Study Question Six } & 49\end{array}$

$\begin{array}{ll}\text { Study Question Seven } & 50\end{array}$

Study Question Eight $\quad 52$

Study Question Nine $\quad 53$

Exploratory Analyses $\quad 54$

CHAPTER FIVE: DISCUSSION 55

Summary of Results and Relationship to Previous Literature 55

$\begin{array}{ll}\text { Exploratory Examinations } & 63\end{array}$

$\begin{array}{ll}\text { Limitations } & 64\end{array}$

Implications for Future Research $\quad 67$

Implications for Prevention and Therapy $\quad 69$

$\begin{array}{ll}\text { Conclusion } & 70\end{array}$

$\begin{array}{ll}\text { REFERENCES } & 72\end{array}$

$\begin{array}{ll}\text { APPENDIXES } & 78\end{array}$

$\begin{array}{ll}\text { VITA } & 106\end{array}$ 


\section{LIST OF TABLES}

Table Page

1. Means, Medians, Standard Deviations for Study Scales 99

2. Number and Percentage of Responses for Items 28 and 29 of the Body Areas Satisfaction subscale 100

3. Number and Percentage of Responses for Author Constructed Items 101

4. Intercorrelations between Variables: Items 28 and 29, Appearance Evaluation subscale, Body Shape Questionnaire 102

5. Intercorrelations between Variables: Author Constructed Items, Appearance Evaluation Subscale, Body Shape Questionnaire 103

6. Intercorrelations between Variables: Items 28 and 29, Appearance Evaluation subscale, Discrepancy Score 104

7. Intercorrelations between Variables: Author Constructed Items, Discrepancy Score 


\title{
THE IDEAL BODY SHAPE OF AFRICAN AMERICAN COLLEGE WOMEN
}

\author{
Rashanta A. Bledman
}

Dr. Laurie Mintz, Dissertation Supervisor

\begin{abstract}
Previous research has examined Black women's body image from a traditional Eurocentric perspective. This has resulted in Black women reporting more positive body image because the research methodology and focus is solely on whether or not these women want to be thin. Rather than focusing on the actual weight on the scales, the overarching goal of this study was to change the focus of body image research with Black women to that of shape and size satisfaction, rather than weight and thinness. Specifically, this study is one of the firsts to examine the ideal body shape of African American women, focusing on level of satisfaction with areas of the body (i.e. the mid and lower torso). Seventy-nine African American women enrolled at the University of Missouri completed an online survey with several instruments designed to measure concerns about body shape and experiences of feeling fat, body image and weight-related concerns, and shape satisfaction. Several regression analyses demonstrated that satisfaction with mid and lower torso significantly influenced body shape satisfaction and overall appearance evaluation. Implications for future research, prevention and counseling are discussed.
\end{abstract}




\section{Chapter One: Introduction}

There is a societal myth that women of color, especially Black women are buffered against dissatisfied or disordered body image and are not susceptible to the pressure that society puts on attaining a specific body shape and size. Indeed, this myth may be the reason why research on body image has historically focused solely on middle to upper class White women. In recent attempts to include Black women into the literature, most researchers have focused on comparing them to their White counterparts, thus, essentially using a Eurocentric standard of beauty (i.e., thinness) to understand the experiences of Black women. This method of research is inherently flawed, as it does not take into account the individual experiences of the Black/African American (AA) woman (The terms Black and African American will continue to be used interchangeably). It is, therefore, important for researchers to begin examining Black women within a cultural context that is relative to them, rather than using an approach and ideal that are not appreciative of their lived experiences.

An underlying assumption of this suggestion is that Black culture is different from mainstream White culture. More specifically, as applicable to this research, the assumption is that Black women may be striving for an ideal shape and size that is different from that of White women. While White women seem primarily concerned with a drive for thinness (i.e., aspiring to a thin body ideal, excessive importance on thinness, and extreme fear of becoming fat; Strigel-Moore, Schreiber, Pike, Wilfley, \& Rodin, 1995), research has found that AA women tend to view body image as a comprehensive phenomenon that includes skin color (Falconer \& Neville, 2000), hair, confidence, overall appearance (Befort, Thomas, Daley, Rhode, \& Ahluwalia, 2008), and style of dress (Rubin, Fitts, \& Becker, 2003). Still, this does not immediately suggest that 
Black women are satisfied with their shape and size. Rather, it may be that Black women are attempting to attain a different ideal--an ideal that has, thus far, not been adequately investigated or understood. Indeed, if these women are unable to attain the body image their culture finds most attractive, they may experience levels of dissatisfaction similar to those of White women who cannot attain their cultural idea of slenderness. To examine this, however, we need to first understand what black women do consider as ideal. Likewise, we need to understand Black women as existing both within their own culture and within the majority White culture.

Black women can be considered bicultural, as they are part of the Black culture but have to survive within the social parameters of White culture. This may make it difficult to understand Black women's experiences and also seems to influence the inconclusive results that are prevalent within the literature on Black women and body image. Some researchers find that Black women have a more positive view of their bodies because of their abilities to accurately predict the shape that men of their culture find most attractive (Molloy \& Herzberger, 1998), have less of a drive or pressure to be thin (Demarest \& Allen, 2000; Gluck and Geliebter, 2002), and live within a culture that allows them to feel accepted at a variety of body shapes and types (Befor et al., 2008). Similarly, other researchers conclude that Black women with high body mass indexes are actually satisfied with specific areas of their bodies (Falconer \& Neville, 2000). In contrast, some researchers find that AA women experience body dissatisfaction and a drive to be thin at similar rates as White women (James, Phelps, \& Bross, 2001), and feel pressure from men, the media and other Black women to attain a certain level of attractiveness (Poran, 2006). Certainly, there is much still be clarified regarding the body image of Black women. 
Some clarification may come from an in-depth examination of various studies. Especially enlightening is research examining how Black women feel about themselves and their attractiveness.

Two studies, in particular, may shed light on how Black women feel about their body image. A study by Poran (2006) reported that Black women experience body dissatisfaction when they notice certain areas, such as their stomach, becoming larger in size. Likewise, in Befort et. al's (2008) study, Black women indicated likes and dislikes about their bodies, with most dislikes focusing on their waist and mid-torso area. Many of the women in Befort et. al's study simultaneously acknowledged a tolerance for larger body sizes, but also described a desire to lose weight. This research demonstrates that Black women's beliefs of attractiveness may rest in the appearance of specific areas of their bodies. Specifically, it may be that Black women want to change the weight and size of certain areas of their bodies (i.e. the mid-torso area), and that they look to those specific zones to determine satisfaction with their appearance. Also enlightening was the finding that for women in the Befort et al. (2008) study, attractiveness and body-esteem did not depend on overall size, but also depended on self-esteem, feeling beautiful as a person, and overall dress, makeup and hair. This finding suggests that Black women may be more concerned with other aspects of their being, rather than just weight, to gauge their feelings of attractiveness. Despite many of these women being overweight, they reported believing that their families were accepting of them, regardless of their weight, while some were even discouraged from losing weight, possibly revealing that this culture does not value a thin body as much as other cultures may.

Thus, it may be that examining Black women's body image from a traditional Eurocentric perspective results in these women reporting more positive body image 
because the research methodology and focus is solely on whether or not these women want to be thin. It seems that "body image" for Black women encompasses more than body weight. Rather than focusing on the actual weight on the scales, it seems appropriate to change the focus of body image research with Black women. In order to effectively gain more than information on the Eurocentric definition of body image, it seems appropriate to focus on the shape that AA women find most attractive. In short, to understand Black women, the focus must be moved from weight/thinness to shape.

Because research has, thus far, been non-existent regarding the shape that AA women strive for, this study will undertake the responsibility of providing more specific information on the ideal shape for Black women. Further, by focusing solely on AA women, rather than using White women as a comparison, this study may provide more insight into the individual lived experience of AA women. Additionally, it is important to provide validation to Black women in acknowledging their individual preferences, rather than assuming that their acceptance of larger body shapes and types is due to what Ard, Greene, Malpeded, and Jefferson (2007) deem a "permissive" culture. The contradicting results of previous literature can possibly be accounted for by examining satisfaction with individual body areas and parts, as well as the influence of such satisfaction on Black women's overall feelings about their bodies. This study thus seeks out to answer the following questions:

1. What do African American (AA) college women report regarding their level of satisfaction with their bodies and shape?

2. What do AA college women say is the ideal body figure for AA women? What is the BMI of these shapes? 
3. What is the level of discrepancy between ideal and actual body shapes of AA college women?

4. Is AA college women's level of satisfaction with their mid and lower torso related to their overall satisfaction with their appearance and their level of concern regarding their body shape?

5. Is AA college women's satisfaction with their mid and low torso related to the discrepancy they report between actual and ideal shapes?

6. Does AA college women's satisfaction with mid and lower torso mediate the relationship between BMI (weight) and overall satisfaction with appearance?

7. Does AA college women's satisfaction with mid and lower torso mediate the relationship between their BMI and their level of concern regarding their body shape?

8. Does African American college women's satisfaction with their stomach and waist, as measured by the author constructed items, mediate the relationship between their BMI and their overall satisfaction with appearance?

9. Does African American college women's satisfaction with their stomach and waist, as measured by the author constructed items, mediate the relationship between their BMI and their level of concern regarding their body shape? It is hypothesized that AA women who are satisfied with their stomach and waist (i.e., flat stomach, small waist) will be satisfied with their bodies regardless of weight, whereas AA women who are dissatisfied with these areas will be dissatisfied regardless of weight. The basis of this hypothesis rests in both the clinical observation of AA women and qualitative studies, such as Befort et al. (2006), which report Black women's discussions on wanting to change certain parts of their bodies and paying particular 
attention to their midsection (i.e. waist). In short, it is hypothesized that body shape may be more important to AA women than body weight. It may be that past research has suffered contradictory results because body shape has not been measured, but instead overall body image satisfaction has been examined. Body dissatisfaction is generally measured as the sum of one's satisfaction with individual parts, but this author argues that for AA women, the sum or average of the parts doesn't equal the whole. Rather than the sum being greater than its parts, where AA women and body satisfaction is concerned, the parts may be greater than the sum. 
Chapter Two: Review of the Literature

The ideal shape for American women encourages the maintenance of a certain level of thinness. There also exists a societal standard suggesting that women are more feminine if they eat little and are petite and delicate (Molloy \& Herzberger, 1998). If women are unable to attain this ideal, the potential to become frustrated and dissatisfied with their looks increases, often resulting in negative body image (Molloy \& Herzberger, 1998). Body image, defined as the mental picture of the body (Thomas, 2001), is a multidimensional construct. It is based on self-perceptions and attitudes regarding physical appearance that can be assessed by examining overall appearance satisfaction or satisfaction with specific body characteristics (Cash, Morrow, Hrabosky, \& Perry, 2004). This author suggests that this popular definition of body image is based on the White women's perspective and does not adequately address the issues of women of color, specifically Black women, who may view body image as a more fluid and flexible phenomenon. When the term body image is used throughout this study, it will be in reference to the previously developed European American definition of body image that does not inclusively capture the cultural context of Black women. It will be clearly noted when this term is used in reference to African American women.

Because there is significant evidence that women are dissatisfied with their bodies (Demarest \& Allen, 2000), body image and its impact on women's self-esteem and perception of themselves has become a topic of interest within the field of psychology. Most body image research has been conducted with White, middle-class college students (Demarest \& Allen, 2000), making it difficult to generalize results to ethnic minority individuals. Specifically, some researchers believe that Black women have been excluded from the literature because of the assumption that these women are members of a culture 
that values and appreciates a larger body (Williamson, 1998). This limitation of the research may also exist because of racial stereotypes and the public's belief that certain mental and physical health problems are solely connected to specific populations, such as the notion that eating disorders and body image disturbances are limited to White women (Gordon, Perez, \& Joiner, 2002). Yanovski (2000) describes the mythology related to eating disorders, body dissatisfaction and race. She suggests that it is important to realize that disorders are not color-blind and, therefore, it is important for clinicians to gain more knowledge regarding how racial and ethnic minorities display dissatisfaction with their bodies and experience symptoms of eating disorders.

Recently, researchers have begun to conduct investigations of body image with Black/African American women, yet there is debate as to whether or not these women are prone to experiencing body dissatisfaction at a significant rate. Some researchers hold the premise that Black women have positive body image, have less of a drive to be thin than White women (Demarest \& Allen, 2000; Lokken, Worthy, Ferraro, \& Attman, 2008; Patel \& Gray, 2001) and are part of a culture that appreciates and finds a larger body attractive (Molloy \& Herzberger, 1998). In contrast, some studies suggest that Black women are striving to maintain a mainstream or majority defined ideal shape and are equally as dissatisfied with their bodies as White women and women of other ethnicities (Demarest \& Allen, 2000; James, Phelps, \& Bross, 2001; Perez \& Joiner, 2003; Poran, 2006; Thomas, 2001;).

Research often compares Black and White women in an attempt to understand how Black women relate to or feel about their weight and bodies (White \& Grilo, 2005; White, Holmaier, Varnado-Sulliva, \& Williamson, 2003). A great deal of research reports that African American women are part of a culture that accepts a larger body 
(Befort, Thomas, Daley, Rhode, \& Ahluwalia, 2006; Ard, Greene, Malpede, \& Jefferson, 2007). Interpretations of these findings have included the idea that Black women are part of a "permissive culture" (Ard, Green, Malpede, \& Jefferson, 2007, p. 38) that focuses more on spirituality and personal style rather than informing these women of what it means to maintain a healthy body size (Ard, Greene, Malpede, \& Jefferson, 2007). In the view of this author, these assumptions are inherently racist. First, the notion of comparing Black and White women is flawed, in that it assumes that White women are the norm at best, and correct at worst. Further insulting are conclusions building upon such assumptions of White normalcy and standard setting, and which thus do not take into account the idea that the African American culture may hold a different standard of beauty that does not mirror that of the dominant White culture's (Gordon, Perez, \& Joiner, 2002).

Such assumptions and conclusions are harmful to Black women. Specifically, the idea that Black women are protected from experiencing disturbances in their eating and images of themselves may limit the research on African American women's body shape satisfaction. Further, these assumptions are detrimental because they may impede professionals from accurately diagnosing or recognizing symptoms and concerns related to body dissatisfaction and eating disorders among minority women (Gordon, Perez, \& Joiner, 2002).

Because of the increased importance in determining how body image satisfaction impacts women's lives and in order to gain a greater understanding of the previous research findings, this chapter will focus on body image within the African American female community. Because of the previously stated myth and assumption of protection from body image disturbances, a review of the proposed protective factors will be given. 
Additionally, the impact of the media on Black women's body concerns will be discussed. This literature review will then focus on the body image literature pertaining specifically to Black women and their level of satisfaction or dissatisfaction with their bodies. Some of these studies compare Black women to White women or Black women to other women of color. This is often done in a search to determine the reasons Black women are more or less satisfied with their bodies than other women. In contrast, some studies, both qualitative and quantitative, focus solely on Black women. Despite their focus, a significant amount of these studies continue to compare Black women to other, often White, women. All of the following studies continue this trend and also fall prey to the flaw of using a narrow, Eurocentric definition of body image, consequently failing to shed light on the possible importance of body shape in the Black female community. Therefore, a thorough review of the only two existing studies focused on Black women and shape are included.

\section{Possible Protective Factors}

Some studies have found that Black women's positive body image is a result of being buffered or being immune to experiences of body dissatisfaction (Lokken, Worthy, Ferraro, \& Attmann, 2008). Molloy and Herzberger (1998) investigated the varying levels of self-esteem and body image satisfaction of a sample of African American and Caucasian women. Participants in this study completed the Body Esteem Scale (Franzoi \& Shields, 1984) and the Body Size Drawings Inventory (Silberstein, Striegel-Moore, Timko, \& Rodin, 1988) to specifically assess body image concerns. The researchers' hypotheses were supported in that African American women reported more positive selfesteem and body image than White women. They also reported that Black women held beliefs and perceptions that men of their race are attracted to a larger body type. Molloy 
and Herzberger's study suggests that assumptions that African American women are protected against negative body image and low self-esteem are indeed supported. As well, it may be that Black women's perceptions of Black men's preferences may allow these women to be more satisfied with their bodies at larger sizes and shapes.

There is also a possibility that there exists different eating and body disturbances that are culture specific and manifested and expressed differently, with some being more prevalent among White women and others occurring equally across ethnicities (White \& Grilo, 2005). A belief also exists that African American and Caucasian women are part of two different cultures that result in different standards of beauty, with Black women reportedly having a more flexible and fluid perception of image, with less emphasis on weight, dieting and a thin body (Molloy \& Herzberger, 1998). This different standard lends support to the idea that African American women are protected, or buffered, from negative body image because there is supposedly less of an emphasis on being thin within the Black culture. This emphasis is based on researchers' assumptions that African American women base their idea of beauty and ideal body shape on the perceptions that men of their race prefer and appreciate larger women (Molloy \& Herzberger, 1998). Some studies have investigated this idea and have measured African American women's accuracy of perceptions in determining the body type to which African American men are attracted (Demarest \& Allen, 2000; Patel \& Gray, 2001), concluding that African American women are able to accurately predict the thinness preferred by men of their race.

Another proposed protective factor is said to be that Black women, in relation to their White counterparts, tend to describe their gender role orientation in masculine or more androgynous traits, therefore, as a result, embodying more positive self-esteem and 
body image, and satisfaction with their sexuality (Molloy \& Herzberger, 1998). This factor is in direct contrast with the societal standard that suggests women are more delicate if they are thinner. It may be that because Black women are thought to define themselves in a more global perspective, they are less likely to be prone to standards that define femininity or womanhood.

Acculturation level is often cited as a protective factor, as it is said that African American women that are strongly identified with the Black culture and interact predominately with other African American individuals tend not to be susceptible to White standards of beauty related to body shape. Some research states that if African American women are more identified with the White culture, possibly as a result of social mobility, they may be more susceptible to pressures to be thin (Molloy \& Herzberger, 1998).

\section{Impact of Media}

Literature is beginning to conclude that the media has played a significant role in shaping Black women's views of their image. Images of women in the media are not only thinner than the actual female population, but they are also thinner than criteria for specific eating disorders and thinner than images of women in the past (Schooler, Ward, Merriwether, \& Caruthers, 2004). The media is very accessible and important in our society, sometimes shaping who and what adolescents want to be (Botta, 2000). In measuring the typical daily use of various forms of media (i.e. television, videotapes, computer and video games, and music videos), Borzekowski, Robinson, and Killen (2000) found that Black teenage girls self-report exposure to 55.2 hours a week of media, specifically television and music videos, lending support to findings that Black girls watch more television than White girls (Botta, 2000; Schooler, Ward, Merriwether, \& 
Caruthers, 2004) and their Asian and Latina counterparts (Borzekowski, Robinson, \& Kellen, 2000). This suggests that Black girls may experience more exposure to media images than girls of other races/ethnicities, which has the potential to further influence feelings about their bodies. In reviewing past studies, Botta (2000) discusses the impact that television has had on body image disturbances in women and suggests that the media encourages the thin cultural ideal by limiting the range of images that are portrayed on television. Although Botta (2000) reports that Black adolescent girls in her study experienced less body dissatisfaction and had a larger personal ideal size, they engaged in similar levels of bulimic behaviors and drive for thinness as White girls. Further, girls who idealized television images held a stronger drive for thinness, with Black girls who idealized thinness the most reporting more disordered behaviors than Whites (Botta, 2000).

The type of media viewed seems to play a role in determining to what extent Black women are affected by body image disturbances. Schooler, Ward, Merriwether, and Caruthers (2004) report that Black women who viewed programs with predominately Black casts experienced a protective effect on their body image, suggesting that appearance related programming on Black-oriented shows may differ from those of White-oriented shows. Despite this finding, the researchers used retrospective reporting of how often women watched television with predominately Black casts (Family Matters, Fresh Prince of Bel-Air, Hangin' with Mr. Cooper, Living Single, Martin, Sister Sister, Jamie Foxx, Moesha, The Smart Guy, and The Wayans Brothers). This serves as a limitation as the authors appear to draw conclusions based on viewing of programs at least four years prior. Not only does this suggest a limitation, but it also lends support to 
the limited number of programs with Black casts on television as approximately seven of the ten shows are no longer in production, with three in syndication.

Like the body image and body shape literature, there still is a parity of research on the role of race in the media, despite findings that media does play a significant role in the body image development of Black and White women. As Black women typically report fewer body image disturbances in attitude, affect, and behavior (Schooler, Ward, Merriwether, \& Caruthers, 2004), individuals may believe that the media does not play a significant role in influencing Black women's views of their bodies. Consequently, the media has consistently limited its presentation of women of color, while continually presenting the societal ideals of beauty: thin and White. Research on the media and its influences often use social comparison as a theoretical framework, often aiming to determine whether Black women compare themselves to White idealized media images or with Black images. Frisby (2004) found that when viewing advertisements with White women, Black female college students most often made comments regarding the White woman's attractiveness, but that such viewing did not result in changes in self-esteem, self-concept or mood. Further investigation in Frisby's study (2004) indicated that although Black women appeared unaffected by images of White women, exposure to images of Black female models caused a negative effect for Black women that were already dissatisfied with their bodies.

\section{Black Women's Satisfaction with Their Bodies}

As previously mentioned, researchers often compare Black and White women when studying body image dissatisfaction. These comparisons often result in contradictory results, some of which state that Black women are satisfied with their 
bodies, and some of which state that Black women are dissatisfied with their bodies. This section will review studies finding that Black women are satisfied with their bodies.

In their study, Cash, Morrow, Hrabosky, and Perry (2004) set out to determine whether body image changes over time and to what extent individuals are psychologically invested in their appearance. A 19-year longitudinal study with mainly Black, White, Asian, and Hispanic individuals from a moderately large mid-Atlantic university allowed these researchers to investigate overall view of appearance, assess fat anxiety, measure cognitive and behavioral investment in appearance, and measure satisfaction with physical characteristics using the Appearance Evaluation, Body Areas Satisfaction, Overweight Preoccupation, and Appearance Orientation subscales of the widely used Multidimensional Body-Self Relations Questionnaire (MBSRQ; 2000). Although results indicated that BMI increased over time for Black women, these women reported more positive global appearance evaluation, less overweight preoccupation and greater appearance investment than their non-Black counterparts. Likewise, Black women in more recent cohorts of the study reported a more favorable body image than non-Black women, despite reporting higher BMIs than the non-Black women with whom they were being compared. Although these Black women were not satisfied with their weight, they were satisfied with their overall body appearance.

Miller, Gleaves, Hirsch, Green, Snow, and Corbett (2000) similarly found that Black women appeared more satisfied with their bodies than White women. Their analyses indicated that appearance was equally important to all women, as evidenced by similar scores on the Appearance Orientation subscale of the MBSRQ, but Black women scored higher than White women on Appearance Evaluation and Body Area Satisfaction 
subscales, rated themselves higher on the Sexual Attractiveness subscale and endorsed more self-esteem regarding their weight.

Drawing similar conclusions, because of African American women's lower scores on the overeating and weight/dieting subscales used in their study, Gluck and Geliebter (2002) stated that Black women are satisfied with their bodies. These researchers further concluded that the African American/Black culture puts less pressure on Black women to be thin, allowing them to experience less body discrepancy. In further support, Lokken, Worthy, Ferraro, and Attmann (2008) report that although Black women had higher BMIs than their White counterparts, they endorsed lower levels of sociocultural influences on mainstream standards of appearance and were less likely to internalize the thin standard.

Bolstering these concusions further, Snooks and Hall (2002) found that Black women in their study were satisfied with their body image. This study examined the role of race in the relationship between weight, body image, and self-esteem. These authors compared Black, White, and Mexican American women's body mass indexes and their identification of real and ideal body sizes and found that Black women were the most satisfied with their body sizes and shapes. Although African American women were heavier than White and Mexican women, they exhibited greater congruency between their real and ideal body sizes. Also, despite being heavier, Black women showed no difference in self-esteem, as measured by the Texas Social Behavior Inventory, from White and Mexican women.

In hopes of further understanding the positive images Black women have of their bodies, Cash, Morrow, Hrabosky, and Perry (2004) performed a cross-sectional study with undergraduate students at a moderately large mid-Atlantic public university. 
Participants were given four subscales of the Multidimensional Body-Self Relations

Questionnaire: Appearance Evaluation, Body Areas Satisfaction, Overweight

Preoccupation, and Appearance Orientation. These researchers found that Black women had a more favorable overall body image, less overweight preoccupation, and a higher lower torso satisfaction in comparison to White women.

Similarly, Gluck and Geliebter (2002) studied undergraduate students to determine whether a relationship exists between race/ethnicity and body image and eating disorders. These researchers examined 108 White and 46 Black female students from a northeast Ivy League University. Participants' body mass index was calculated through their recorded height and weight. They were then given the Stunkard Figure Rating Scale (Stunkard, Sorenson, \& Schulsinger, 1983) and asked to indicate their current and ideal body sizes. After controlling for BMI, results indicated that African American women chose a similar ideal as White and Caucasian women, yet experienced less cultural pressure to attain this idea. Lastly, these authors found that the African American women in this study experienced less discrepancy between their current and ideal sizes.

In short, many studies have found that Black women are satisfied with their bodies, both in general and in comparison to White and other ethnic minority women. Another sentence or two summary if you can think of it here. Such studies, however, are in contrast to those finding that Black women are dissatisfied with their bodies, which are detailed in the section below.

\section{Black Women's Dissatisfaction with Their Bodies}

The influence and internalization of the thin ideal has been linked to the development of body dissatisfaction, dieting behaviors, negative affect and eating disorders in women, as the ideal that is internalized is often one that is virtually 
unattainable (Thompson \& Stice, 2001). The thin ideal has also been linked to selfstarvation, binge-eating and purging (Williamson, 1998). It is suggested that because Black women and other women of color are exposed to similar stimuli as White women, they may not be fully immune to and protected from internalizing the drive for thinness and therefore developing body dissatisfaction. It is possible that, despite membership in the culture of an ethnic group that accepts a larger female body, white American standards are still influential (Gordon, Perez, \& Joiner, Jr., 2001).

Some studies suggest that Black women are equally as dissatisfied with their bodies when compared to White women or women of other ethnicities (Perez \& Joiner, Jr., 2003; Thomas, 2001; Demarest \& Allen, 2000; Poran, 2006; James, Phelps, \& Bross, 2001) and are striving to maintain the thin ideal body shape. Despite research suggesting that African American women are satisfied with their bodies, there are still findings that reveal that African American women prefer smaller body images and chose the same ideal body image as White women, with a BMI of approximately 21 (Ard, Greene, Malpede, \& Jefferson, 2007). When asked how being a Black women affects their weight, participants in Ard, Greene, Malpede, and Jefferson's 2007 study revealed five themes: food preparation, food selection, lack of exercise, risk of chronic disease, and multiple roles that make healthy food choices difficult to make. Although these themes were common among the Black women in the study, the White women that participated shared no common themes with the African American women, suggesting that Black and White women may have different thoughts regarding weight, body and the influences on these two factors. Interestingly, many of the obese Black women in this study believed that they were only overweight. 
Demarest and Allen (2000) used figure rating drawings to examine the current and ideal body image figures of African American, Hispanic and Caucasian individuals. Each figure on the figure rating scale corresponded with a number from 10-90, with 10 being the thinnest. Each participant was asked to choose their current and ideal figures on a same-sex figure scale, and then identify what he or she thought to be the most attractive to the opposite sex. They were lastly asked to determine what they found to be the most attractive figure of the opposite sex. Although body image dissatisfaction was greatest among women, in comparison to men, the African American women seemed to have greater body esteem than other groups because they were better able to accurately perceive the body shape that was preferred most by men. Despite this accuracy in perception, the Black women in this study were not more satisfied than Hispanic and White women. These African American participants actually wanted to be thinner and seemed to have an understanding of how thin men wanted them to be.

Similarly, Perez and Joiner (2003) found that Black women were dissatisfied with their bodies, yet in a different fashion than White women. These researchers compared Black and White undergraduate women on responses to the Body Image Scale (Stunkard, Sorenson, \& Schulsinger, 1983) and Eating Disorder Inventory (Garner, Olmsted, \& Polivy, 1983). Their results indicated that Black women reported smaller differences between their current and ideal body image than did White women, but the larger Black women perceived their body to be, the more discrepant their score between current and ideal. Another indication of dissatisfaction was Black women's report of being underweight, while simultaneously indicating a larger body as their ideal size. In other words, for some Black women in this study, their negative body image resulted from weighing less than they wanted to. This suggests that Black women may be striving for a 
different ideal, and becoming dissatisfied when they are unable to achieve it. According to the results of this, and other similar studies, Black women are therefore not buffered from low body satisfaction and disordered eating behaviors.

Although a great deal of research compares Black and White women, some studies have begun to compare Black women to other women of color. Specifically, Hrabosky and Grilo (2007) used a variety of instruments, including the Body Shape Questionnaire (1987), to examine the eating and body image concerns of Black and Hispanic women. Although these authors recognize that past research has indicated a lower incidence of concern for eating and weight related disorders in ethnic minority women, their study's results indicate both Black and Hispanic women reported comparable levels of body image and eating related concerns. They also reported little difference in their depressive affect related to their concerns. Likewise, women in both groups indicated experiences of being mocked because of their weight and shape. Interestingly for these women, body mass index did not predict body image concerns, suggesting that for women of color, increases in body mass index may not have a linear relationship with body image dissatisfaction. It may also be that eating and weight related concerns are manifested differently in women of color and are a result of predictors that are specific to their group. White and Grilo (2005) found that body image concerns were present in their sample of White, Black, and Latina women, yet the predictors of these women's concerns varied by ethnic group. Although these researchers determined that White women were more dissatisfied with their bodies than Black women, they reported that the dissatisfaction that Black women do experience is a result of negative self-esteem, peer insecurity and anxiety. 
Rather than using methods of comparison with other groups of women, some researchers have focused solely on Black women in their attempts to determine bodyimage satisfaction and shape concerns. This approach allowed Thomas (2001) to use a sample of 102 African American women in a metropolitan area to determine the level of body satisfaction they experience. The participants in this study completed a questionnaire that asked them to indicate their satisfaction with their bodies on a Likerttype scale ranging from very unhappy (1) to very happy (5). They were also asked to indicate perceptions of their body size (i.e. too fat, too thin, just right) and height (too, short, too tall, just right). Only $34.7 \%$ of the women were happy or somewhat happy, while almost $55 \%$ of the women expressed feelings that ranged from very unhappy to somewhat unhappy with their bodies.

James, Phelps, and Bross (2001) discussed the assumption of Black women's immunity from eating disorders and body image disturbances. In order to further investigate this idea, these researchers evaluated ninety-five African American undergraduate women at a predominately White institution to determine their level of body dissatisfaction, drive for thinness, and self-concept (i.e. physical, social, academic, and personal competence). By using the Body Dissatisfaction and Drive for Thinness subscales of the Eating Disorders Inventory-2 (Garner, 1991) and the Academic, Competence, Physical and Social subscales of the Multidimensional Self-Concept Scale (Bracken, 1992). James, Phelps, and Bross (2001) determined that this sample of Black undergraduate women had body dissatisfaction and drive for thinness scores that are similar to those of their white counterparts. These researchers concluded that Black women's dissatisfaction was correlated with both a desire to be thin and low level of positive affirmation for their physical appearance. It may be that African American 
women could benefit from receiving positive affirmation regarding their appearance and encouragement to avoid accepting a thin cultural ideal.

Just as the media plays a significant influence on body image, the perceived preferences of Black men may also be an influential contributing factor to how Black women feel about their bodies. Although some research has found that Black men prefer larger bodies, Webb, Looby, and Fults-McMurtery (2004) report that African American men are beginning to find smaller body figures as more attractive, suggesting that these men may begin to pressure Black women to maintain a thin body to be considered attractive to men of their race. Interestingly, Demarest and Allen (2000) found when presented with the opportunity to view a figure rating scale, Black women picked ideal body figures that were larger than the figures they believed were most attractive to Black men. These differences may further influence Black women's struggles with body image, suggesting that they may believe men of their race find them attractive at a lower weight than these women themselves are actually comfortable with.

In another study pointing to Black women's dissatisfaction with their bodies, Thomas (2001) found that Black women in her study reported an ideal weight that was lower than their actual weight. This finding likely stems from these women accepting or internalizing a thin ideal body shape.. As previously mentioned, the thin ideal has been investigated in regards to its influence on the body image satisfaction of Black women. James, Phelps, and Bross (2001) found that Black women wanted to be thinner, evidenced by their comparable scores to White women on measures of body dissatisfaction and drive for thinness. These authors used the Body Dissatisfaction and Drive for Thinness subscales of the Eating Disorders Inventory-2 (Garner, 1991) and the Academic, Competence, Physical and Social subscales of the Multidimensional Self- 
Concept Scale (Bracken, 1992) to determine the level of body dissatisfaction and drive for thinness in their African American female sample. Because the Black women were students at a predominately White college institution, James, Phelps, and Bross (2001) suggest that Black women may experience body dissatisfaction as a result of internalization of the White thin ideal.

Despite the previously explained support for the idea of protection for Black women, Poran's (2006) extensive qualitative study regarding protective factors suggests that these women do not possess high body esteem and indicate no significant comfort with their bodies. Further, Poran considers it myth that the Black culture supposedly protects and shields Black women from experiencing body image dissatisfaction. In her study, Poran (2006) found that most of the supposed protective factors that have been mentioned in the research often act as sources of pressure. Specifically, analyses revealed several themes: 1) critique of thinness as part of the beauty standard in American; 2) the perception of pressures through different standards held by men of diverse ethnic backgrounds; 3) competition among young women, and 4) the perception of being misrepresented and essentialized by dominant cultural images of women. Poran's themes reveal that many of the factors that are supposed to protect Black women actually contribute to various pressures they experience in regards to their bodies.

Black Women's Satisfaction with Specific Body Areas

As can be ascertained, research on African American women's body satisfaction has resulted in contradictory results. Surprisingly, these contradictory findings are sometimes apparent within one study. Falconer and Neville (2000) investigated the body mass satisfaction of 124 African American college women. Their research was conducted by using the African Self-Consciousness scale (Baldwin \& Bell, 1985) the 
Skin Color Satisfaction Scale developed by Falconer and Neville for the purposes of their study, the Appearance Evaluation, Appearance Orientation, and Body Areas Satisfaction subscales of the Multidimensional Body-Self Relations Questionnaire (2000). Falconer and Neville (2000) found that those women satisfied with their skin color were also more satisfied with their body shape. Although women with higher body mass index categories were more dissatisfied with their overall bodies, they exhibited a higher satisfaction with specific areas of their bodies. The researchers suggest that this contradiction may be related to the African American culture placing a higher value on having a curvier body with larger buttocks and hips. It may be that heavier Black women are able to find satisfaction with specific body areas, which may assist in mediating the relationship between their body mass index and overall body image and shape satisfaction.

In contrast to Falconer and Neville (2000), Befort, Thomas, Daley, Rhode, and Ahluwalia (2008) developed a qualitative study in which they examined the perceptions and beliefs about body size and weight among African American women. Results from the six focus groups in the study determined that the women acknowledged being overweight and were dissatisfied with their weight. Yet, they did not believe that attractiveness, self-esteem or health were dependent on body size. Further, these women reported believing that widely accepted height and weight charts used to calculate body mass index are inappropriate for African American women, as the participants believed that Black women have larger frames. As well, the women in this research study reported that their body satisfaction was actually dependent on certain body parts, specifically their waist. Although these women reported that they were not at the weight or shape they preferred, they reported that family and friends accepted them regardless of 
their body size and prioritized the needs of their family over concern for their bodies. Also, though these women acknowledged their own culture's acceptance of a larger body and beliefs that size did not influence attractiveness and happiness, they did conclude that they were concerned with their weight and often self-conscious about their bodies. Although these women did acknowledge a need or desire to lose weight, their concern appeared to be on certain body parts, specifically their waist. This focus lends support to the idea that Black women hold a specific body image in mind that may involve being more concerned with the shape of the mid-torso, rather than worry over the appearance of the entire body.

\section{Purpose of Current Investigation}

The contradictory results of the previously reviewed studies are interesting. They suggest that the particular aspects of body image within the African American female community have yet to be adequately and appropriately measured. It seems that at higher weights, Black women may be dissatisfied with their overall body image, but may also be simultaneously satisfied and comfortable with certain aspects of their bodies. These studies also suggest that specific areas of the body, such as the buttocks, hips, and waist, may be more important for African American women in determining their level of satisfaction. Therefore, this study will continue the process of conducting research to understand African American women's body concerns. 


\section{Chapter Three: Method}

This chapter will be divided into four subsections. The first subsection will describe the participants of this study. The second subsection will detail the psychometric properties of the following instruments that were used: the Multidimensional Body-Self Relations Questionnaire (MBSRQ) was used to assess body image and weight-related concerns; the Body Shape Questionnaire (BSQ) was used to measure body shape concerns and feelings of being fat; and the Reese Figure Rating Scale was used to determine ideal and current body shape and size. In the third subsection, the procedures will be outlined. Finally, the fourth subsection will discuss the statistical analyses.

\section{Participants}

Participants were 79 undergraduate women from a predominately White, large public Midwestern University. These participants were among 90 individuals who originally logged on to complete the study. Eleven participants were eliminated for a variety of reasons. One individual was not included due to indicating both an ethnicity of Caucasian a gender of male on the demographic questionnaire. A second participant was excluded due to indicating a graduate student status. Seven individuals from this same large Midwestern University logged on but did not complete any instruments in the survey. Finally, two individuals were eliminated due to being from a different university than the rest of the participants (i.e., a small, predominately Black private Midwest school).

Of the 79 participants, 70 completed the demographic questionnaire, and therefore the following descriptive statistics are based on their information. All participants were African American/Black women. Ages ranged from 17 to 25 (M=20.01, SD=1.79), with 
$59 \%$ of individuals indicating they were single, $14 \%$ dating casually, $20 \%$ dating seriously, $6 \%$ married or in a committed relationship, and $1 \%$ engaged to be married. Ninety-five percent of participants considered themselves to be heterosexual, with the remaining 5\% identifying as bisexual. The average weight of participants was $157 \mathrm{lbs}$, with an average height of 65 inches (i.e., 5'5'). Furthermore, 2\% considered themselves to be upper class, $16 \%$ upper middle class, $50 \%$ middle class, and $32 \%$ working class. Lastly, $89 \%$ were Christian and the remaining $11 \%$ indicated being non-religious.

\section{Instruments}

\section{Multidimensional Body-Self Relations Questionnaire-Appearance Scales} (MBSRQ-AS; Cash, 2000). The MBSRQ (Brown, Cash and Mikulka, 1990) is a multidimensional assessment of body image and weight-related concerns in adults and adolescents 15 years and older. It is comprised of several subscales: Appearance Evaluation, Appearance Orientation, Fitness Evaluation, Fitness Orientation, Health Evaluation, Health Orientation, Illness Orientation, Body Areas Satisfaction, Overweight Preoccupation, and Self-Classified Weight. A shortened version, the 34-item MBSRQAS (Cash, 2000) focuses solely on appearance-related body satisfaction. This version was used for the purposes of this study. The MBSRQ-AS was developed through utilizing a sample consisting of 2,052 individuals (988 men and 1,064 women) that participated in a national survey on body image. These individuals were randomly sampled from the Northeast, South, Midwest and Eastern regions of the United States, and included 91\% White individuals and 55\% college or graduate educated individuals.

The MBSRQ-AS includes the following scales: Appearance Evaluation, Appearance Orientation, Overweight Preoccupation, Body Areas Satisfaction Scale, and Self-Classified Weight. Questions on the first three scales (Appearance Evaluation, 
Appearance Orientation, and Overweight Preoccupation) are answered using a five-point Likert-type scale format with responses ranging from (1) strongly disagree to (5) strongly agree, with the exception of two items on the Overweight Preoccupation subscale in which the anchors differ, although the response options still range from one to five.. Scores on each of these three subscales are the average for those on the scale, with scores thus ranging from one to five. More specifically, the Appearance Evaluation subscale consists of seven items that assess feelings of physical attractiveness. High scores indicate positive feelings and satisfaction with appearance, while lower scores indicate unhappiness with appearance. This subscale has an internal consistency of .88 and a testretest correlation of .91. Sample questions include "I like my looks just the way they are" and "I like the way my clothes fit me." The Appearance Orientation subscale consists of 12 items that assess cognitive and behavioral investment in one's appearance. High scores indicate that an individual places significant importance on how they look, pays attention to their appearance, and engages in extensive self-grooming behaviors. In contrast, those with lower scores tend to exhibit apathy toward their appearance and do not believe that their physical appearance is important. This subscale has an internal consistency of .85 and a test-retest reliability coefficient of .90 . Sample questions include "I don't care what people think of my appearance" and "I am always trying to improve my physical appearance." The Overweight Preoccupation subscale consists of four items that assesses fat anxiety, weight vigilance, dieting and eating restraint. The first three items are scored using a five-point Likert-type scale as described above (i.e. with responses ranging from (1) strongly disagree to (5) strongly agree), while the fourth item requires individuals to indicate their frequency of participating in fasting or crash diets, with responses ranging from never to very often. High scores suggest an individual that is 
more preoccupied by their weight, while lower scores suggest less anxiety regarding with one's weight. This subscale has an internal consistency of .76 and test-retest reliability of .89. Sample questions include "I constantly worry about being or becoming fat" and "I am very conscious of even small changes in my weight."

The Body Areas Satisfaction subscale consists of nine items that assess level of satisfaction with various body parts, areas or aspects. These nine items are: 1) face (facial features and complexion); 2) hair (color, thickness, texture); 3) height; 4) weight; 5) upper torso (chests or breasts, shoulders, and arms); 6) mid-torso (waist and stomach); 7) lower torso (butt, hips, thighs, legs); 8) muscularity/muscle tone; and 9) overall appearance. These nine items are answered using a five-point Likert-type scale format with responses ranging from (1) Very Dissatisfied to (5) Very Satisfied. Scores on this subscale are the average for those on the scale, with scores thus ranging from one to five. High scores thus indicate content with most areas of the body, while lower scores indicate unhappiness with size or appearance of several areas. This subscale has an internal consistency of .73 and a test-retest reliability of .74 .

The final subscale, Self-Classified Weight, consists of two items that require individuals to indicate what weight category they and others consider them to be, with response options ranging from (1) very underweight to (5) very overweight. Scores are an average of the two items, and thus higher scores indicate more self and otherperceived views of being overweight. This subscale has an internal consistency of .89 and test-retest reliability of .74 .

The MBSRQ is a widely used instrument in the assessment of body image. Most pertinent for this study, this instrument has been successfully used in various studies with African American women (Cash, Morrow, Hrabosky, \& Perry, 2004; Harris, 1995; 
Miller, Gleaves, Hirsch, Green, Snow, \& Corbett, 2000; Rucker \& Cash, 1992; Smith, Thompson, Raczynski, \& Hilner, 1997). The MBSRQ can be found in Appendix A.

The Body Shape Questionnaire (BSQ; Cooper, Taylor, Cooper, and Fairburn, 1987). The BSQ is a 34-item self-report instrument that measures concerns about (i.e., preoccupation with) body shape. Responses are provided on a six point Likert-type scale ranging from (1) never to (6) always. Thus, possible scores range from 34 - 204, with high scores indicating more concern and lower scores indicating less concern with body shape. The BSQ asks participants to focus on their feelings regarding their appearance over the past four weeks. Example items include, "Have you been afraid that you might become fat (fatter)?" and "Has worry about your shape made you diet?"

Cooper, Taylor, Cooper, and Fairburn (1987) developed the BSQ by sampling four groups of women, totaling a number of 573 individuals: patients with bulimia nervosa, family planning clinic attendees, occupational therapy students, and female university undergraduate students. Cooper et al. reported that the BSQ correlated with both the Body Dissatisfaction subscale of the Eating Disorder Inventory (Garner, Olmstead, \& Polivy, 1983) and the Eating Attitudes Test (Garner \& Garfinkel, 1979). Cooper and colleagues (1987) also compared two groups of non-clinical women: one group of 95 women who were dieting to lose weight and rated slimness and fear of fatness as moderately or markedly important, and another group of 79 women who were not dieting to lose weight and considered slimness and fear of fatness as not at all or slightly important. In comparing these two groups, Cooper et al. found that their scores on the BSQ were significantly different. Similarly, Cooper and colleagues compared a group of ten women classified as being probable cases for bulimia nervosa with 316 women who did not meet any clinical criteria for bulimia. The women with probable 
bulimia evidenced the type of preoccupation with body shape that is indicative of eating disorders. Likewise, the two groups (probable bulimia; no eating disorder) evidenced significant differences in their BSQ scores, further demonstrating the validity of the BSQ. Additional validity of the BSQ was provided by Rosen, Jones, Ramirez, and Waxman (1996), who reported correlations between scores on the BSQ and scores on the Appearance Orientation, Appearance Evaluation, and Body Areas Satisfaction subscales of the MBSRQ (Cash, 2000). Highly pertinent to this study, past research has used the BSQ with Black women, with two past studies reporting the BSQ to have an internal consistency (i.e., Cronbach alpha) of .98 (Schooler, Ward, Merriwether, \& Caruthers, 2004; Hrabosky \& Grilo, 2006). The BSQ can be found in Appendix B.

Reese Figure Rating Scale (Patt, Lane, Finney, Yane, \& Becker, 2002). The Reese Figure Rating Scale was developed because no appropriate previous figure rating scales for Black women existed. Because these authors felt that previous scales were geared toward White women (i.e., Stunkard Rating Scale; Stunkard, Sorenson, \& Schulsinger, 1983), contained too much nudity (i.e., Williamson Figure Rating Scale; Williamson, 1990) or were lacking in important details in Black women's body forms (i.e., Anderson Figure Rating Scale; Anderson, Janes, \& Phillips, 1997), this scale was developed from randomly selected photos of Black women falling into varying Body Mass Index (BMI) categories.

The Reese Figure Rating Scale consists of nine silhouette drawings indicating increasing body sizes, with BMIs ranging from 21.5 to 44.3. Similar to other figure rating scales, when developing this scale, the authors considered normal weight as a BMI $<25$, overweight a BMI of 25-29 and obese a BMI of 30-39. Based on focus group feedback, the differences in the nine images of the scale were developed through 
increasing the BMI of the image, paying particular attention to the thighs, abdomen and breasts. Each image has a corresponding number, with one being the largest, and nine being the smallest. When using this scale, a discrepancy score can be calculated by subtracting the number that corresponds to the figure indicated as an individual's ideal size from the number corresponding to the figure indicated as their current size (Lynch, Liu, Spring, Hankinson, Wei, \& Greenland, 2007).

In developing this scale, Patt et al. (2002) queried a sample of 50 churchgoing Black women, over the age of 40 , recruited during general health screenings at church. These women were asked to view the Reese Scale and three other Figure Rating Scales (Anderson, Janes, \& Phillips, 1997; Stunkard, Sorenson, \& Schulsinger, 1983;

Williamson, 1990) and to indicate the scale they believed best portrayed the body images of themselves and other Black women. The Reese scale was preferred by the overall group as being representative of Black women's shapes. The Reese scale was also found to have accurate scaling properties when compared to other figure rating scales. Specifically, the average BMI of each figure is comparable across scales and all four scales correlated to the actual BMI of the women (i.e. Pearson correlation coefficient range of -.70 to $-.75, \mathrm{p}<.0001)$.

For the purposes of this study, participants were asked to choose the figure that approximates her current shape and the figure that approximates her ideal shape, and discrepancy scores were calculated. The Reese Figure Rating Scale can be found in Appendix C.

In order to assess for reliability, an invitation to complete a follow-up administration of the Reese Figure Rating Scale was emailed to 62 participants who completed the original survey. A total of 22 individuals completed the follow-up 
administration of the Reese Figure Rating Scale. A paired t-test was run to determine the test-retest reliability. This t-test, with a p value of 0.0812 , was insignificant, indicating that the Reese Figure Rating Scale was a reliable measure in this study. Specific to the test-retest data set, the average actual size indicated on the first administration was 6.43 , while the average actual size indicated on the second administration was 6.86 .

Furthermore, the correlation coefficient between actual body size on the first and second administration was -.058 , with a $95 \%$ confidence level. The average ideal size indicated on the first administration was 7.50, while the second administration for test-retest purposes was 7.18. The correlation coefficient between ideal body size on the first and second administration was .189 , with a $95 \%$ confidence level.

Author Constructed Items. Because of the lack of instruments developed to effectively assess shape satisfaction in African American women, author constructed items were also included. Specifically, five items were presented to participants, with responses gathered using a five-point Likert-type scale format with responses ranging from (1) Strongly Disagree to (5) Strongly Agree. The five items are: 1) I am satisfied with my body shape, 2) I am satisfied with the flatness of my stomach, 3) I am satisfied with the narrowness of my waist, 4) As long as my stomach is flat, my weight does not matter, and, 5) As long as my waist is narrow, my weight does not matter. Finally, the item, "If anything, what would you change about your body shape?" asked for narrative answers. The Author Constructed Items can be found in Appendix D.

Demographic Questionnaire. An author constructed questionnaire was used to gather demographic information regarding the participants. This questionnaire included items regarding the participants' age, sex, gender, race/ethnicity, height, weight, current year in college, sexual orientation, relationship status, and socioeconomic status. Self- 
reported height and weight were used to calculate Body Mass Index (BMI; $\left.\mathrm{kg} / \mathrm{m}^{2}\right)$. The Demographic Questionnaire can be found in Appendix E.

\section{Procedure}

Permission for this study was obtained from the University of Missouri Institutional Review Board. Initially, a request for a list of all African American/Black female undergraduate students was sent to the Registrar Office's Student Administration System at the University of Missouri. The request for this list was not granted, due to necessity to uphold the privacy and confidentiality rights of these women. Recruitment was thus conducted through a variety of sources, as described below.

First, during Fall Semester 2009, a recruitment ad was placed in the MU Info Announcement, a weekly email sent to faculty, students, and staff at the University of Missouri (See Appendix F). Interested participants were instructed to contact the researcher by email, at which time they were sent an email message response providing more specific information about the research study and providing a link to the online survey (See Appendix G).

Second, the University of Missouri Institutional Review Board also granted permission for the researcher to contact the following Black/African American campus organizations with a request to forward the research survey link to their members: Delta Sigma Theta Sorority, Legion of Black Collegians, Legion of Black Collegians Gospel Choir, and the National Association of Black Journalists (See Appendix H).

Third, IRB approved flyers were also posted in the MU Gaines/Oldham Black Culture Center and MU Student Health Center (See Appendix I).

Finally, approval for recruitment at Lincoln University, a historically Black college, in Jefferson City, Missouri was granted. Announcements and distribution of 
research study flyers were passed out a professor in several undergraduate psychology courses (See Appendix I).

Given these various methods of recruitment, response rate for the study cannot be accurately calculated. To illustrate, given that this mass email response went to all members of campus, and that is unknown how many actually read the advertisement, response rate cannot be accurately calculated. Similar shortcomings in ability to calculate response rate apply to the hung flyers as well, for example.

Participants who were interested upon receiving any of these various methods of recruitment were directed to a website to take the study (e.g., the advertisements included the URL). Upon loading the website, all research study participants read an informed consent (See Appendix J). They were informed that, because of the nature of the internet, transmission of data is not secure, but was guaranteed upon the researcher's receipt of data. Participants who agreed to the information given in the informed consent were required to do so by choosing the option to continue and they were then given the survey. Participants who continued were instructed to complete the instruments in the following order: the Multidimensional Body-Self Relations Questionnaire, Body Shape Questionnaire, Reese Figure Rating Scale, author constructed item, and demographic questionnaire. Directions on how to complete each measure were included preceding each instrument. After completing the survey, participants were given a debriefing sheet as well as the contact information for the primary investigator and information on where to seek help for body image and/or eating concerns on campus (See Appendix K). The entire study took approximately 20 minutes to complete. In order to compensate participants for their time, a raffle for two $\$ 50$ gift cards to the campus bookstore was held. Similarly, a raffle for one $\$ 50$ gift certificate was held for the individuals from the 
second university. Two weeks after the final participant completed the online study, an invitation to complete a follow-up administration of the Reese Figure Rating Scale was sent (See Appendix L). This was administered in order to determine the test-retest reliability of this instrument. After completing the follow-up administration, a debriefing form was provided to each participant (See Appendix M).

\section{Statistical Analyses}

In order to adequately discuss the proposed statistical analyses, the research questions and proposed analyses are listed together below:

1. What do African American (AA) college women report regarding their level of satisfaction with their bodies and shape?

a. Means, medians, ranges, and standard deviations on the MBSRQ-AS subscales and on the Body Shape Questionnaire, as well the authorconstructed items, were calculated.

i. Particular attention was paid to both the author constructed items and to the scores on items twenty-eight and twenty-nine of the MBSRQ-AS, located on the Body Areas Satisfaction subscale, as these items examine the level of satisfaction with lower (buttocks, hips, thighs, and legs) and mid (waist and stomach) torso, respectively.

2. What do AA college women say is the ideal body figure for AA women? What is the BMI of these shapes?

a. Responses on the Reese Figure Scale were examined. Specifically the percentage of participants indicating they consider each of the nine figures 
as the ideal figure were calculated, as were the average BMI of the ideal figures.

3. What is the level of discrepancy between ideal and actual body shapes of AA college women?

a. Descriptive statistics (e.g., means, medians) for the Reese Figure Rating Scale were calculated for both the ideal (see question \#2, above) and actual shape indicated by participants.

b. A discrepancy score was calculated by subtracting the number of the participant's ideal from their actual shapes indicated on the (Reese Figure Rating Scale).

4. Is AA college women's level of satisfaction with their mid and lower torso related to their overall satisfaction with their appearance and their level of concern regarding their body shape?

a. Correlation analyses were conducted between the Appearance Evaluation subscale score of the MBSRQ-AS and the following items from the Body Area Satisfaction Scale of the MBSRQ-AS: twenty-eight, twenty-nine, and the mean of these two items.

b. Correlation analyses were conducted between the score on the Body Shape Questionnaire and the following items from the Body Area Satisfaction Scale of the MBSRQ-AS: twenty-eight, twenty-nine, and the mean of these two items.

c. Correlation analyses were conducted between the Appearance Evaluation subscale score of the MBSRQ-AS and the second and third author 
constructed items (I am satisfied with the flatness of my stomach; I am satisfied with the narrowness of my waist).

d. Correlation analyses were conducted between the overall score on the Body Shape Questionnaire and the second and third author constructed items (I am satisfied with the flatness of my stomach; I am satisfied with the narrowness of my waist).

5. Is AA college women's satisfaction with their mid and low torso related to the discrepancy they report between actual and ideal shapes?

a. Correlation analyses were conducted between the discrepancy score obtained from the Reese Figure Rating Scale and the following items from the Body Area Satisfaction Scale of the MBSRQ-AS: twenty-eight, twenty-nine, and the mean of these two items.

b. Correlation analyses were conducted between the discrepancy score obtained from the Reese Figure Rating Scale and the following items from the author constructed items: I am satisfied with the flatness of my stomach; I am satisfied with the narrowness of my waist.

6. Does AA college women's satisfaction with mid and lower torso mediate the relationship between BMI (weight) and overall satisfaction with appearance?

a. Mediation was tested using the procedures outlined by Baron and Kenny (1986), which included conducting four sets of regressions to test the relationship between BMI and overall satisfaction with appearance. Specifically, in the first regression analysis, the average of items twentyeight and twenty-nine of the MBSRQ-AS Body Areas Satisfaction subscale were regressed onto BMI. In the second regression, Appearance 
Evaluation scores of the MBSRQ-AS were regressed onto BMI. In the third regression, MBSRQ-AS Appearance Evaluation scores were regressed onto the mean of items twenty-eight and twenty-nine of the MBSRQ-AS Body Area Satisfaction subscale. Lastly, in the fourth regression, Appearance Evaluation scores of the MBSRQ-AS were regressed onto BMI and the mean of items twenty-eight and twenty-nine of MBSRQ-AS Body Area Satisfaction subscale.

7. Does AA college women's satisfaction with mid and lower torso mediate the relationship between their BMI and their level of concern regarding their body shape?

a. Mediation was tested using the procedures outlined by Baron and Kenny (1986), which included conducting four sets of regressions to test the relationship between BMI and level of concern regarding body shape. Specifically, in the first analysis, the average of items twenty-eight and twenty nine of the MBSRQ-AS Body Areas Satisfaction subscale were regressed onto BMI. In the second regression analysis, BSQ scores were regressed onto BMI. In the third regression analysis, BSQ scores were regressed onto the mean of items twenty-eight and twenty-nine from the MBSRQ-AS Body Area Satisfaction subscale. Finally, in the fourth analysis, BSQ scores were regressed onto BMI and the mean of items twenty-eight and twenty-nine from the MBSRQ-AS Body Area Satisfaction subscale. 
8. Does African American college women's satisfaction with their stomach and waist, as measured by the author constructed items, mediate the relationship between their BMI and their overall satisfaction with appearance?

a. Mediation was tested using the procedures outlined by Baron and Kenny (1986), which included conducting four sets of regressions to test the relationship between BMI and overall satisfaction with appearance.. Specifically, in the first regression analysis, the average of author constructed items two and three were regressed onto BMI. In the second regression analysis, Appearance Evaluation scores of the MBSRQ-AS were regressed onto BMI. In the third regression, Appearance Evaluation scores of the MBSRQ-AS were regressed onto the mean of the second and third author constructed items (I am satisfied with the flatness of my stomach; I am satisfied with the narrowness of my waist). Lastly, in the fourth regression, Appearance Evaluation scores of the MBSRQ-AS were regressed onto BMI and the mean of these two author constructed items.

9. Does African American college women's satisfaction with their stomach and waist, as measured by the author constructed items, mediate the relationship between their BMI and their level of concern with body shape?

a. Mediation was tested using the procedures outlined by Baron and Kenny (1986), which included conducting four sets of regressions to test the relationship between BMI and level of concern with body shape. Specifically, in the first regression analysis, the average of author constructed items two and three were regressed onto BMI. In the second regression analysis, BSQ score were regressed onto BMI. In the third 
regression, BSQ scores were regressed onto the mean of the second and third author constructed items (I am satisfied with the flatness of my stomach; I am satisfied with the narrowness of my waist). Finally, in the fourth regression, BSQ scores were regressed onto BMI and the mean of these two author constructed items (I am satisfied with the flatness of my stomach; I am satisfied with the narrowness of my waist). 
This chapter will describe and summarize the results of the statistical analyses used to evaluate the research questions discussed in previous chapters. It will begin by reporting the results related to how satisfied African American/Black college women are with their bodies and the body shape they indicate to be the most ideal. The difference between these women's ideal and actual body mass indexes will be examined. The relationship between reported satisfaction with mid and lower torso and overall satisfaction with appearance will be examined, as will correlations between mid- and lower-torso satisfaction and the following two variables: 1) concern with body shape; and 2) discrepancy between ideal and actual shape. Lastly, statistical analyses will examine the question of if African American women's satisfaction with their mid/lower torso mediates the relationship between body mass index and: 1) overall satisfaction with appearance; and 2) level of body shape concerns.

\section{Data Cleaning and Screening}

Prior to the main analyses, all data was downloaded and visually examined for accuracy of data entry. Participants who did not complete any of the survey instruments were excluded from the data analysis. For the five participants that only completed the MBSRQ-AS, but no other instruments, they were included only in the analyses that were specific to the MBSRQ-AS. Furthermore, missing items were replaced by the mean of the items on the specific instrument. The number of means replaced for the MBSRQ-AS subscales were as follows: 1) six on the Appearance Evaluation subscale; 2) nine on the Appearance Orientation subscale; 3) five on the Body Areas Satisfaction subscale; and 4) two on the Overweight Preoccupation subscale. All participants completed the Self- 
Classified Weight subscale. Twenty-two means were replaced on the Body Shape Questionnaire.

Study Question \#1: What do African American (AA) college women report regarding their level of satisfaction with their bodies and shape?

To determine how AA women in this study feel about their overall bodies and shape, the means, medians, and standard deviations for each scale of the MBSRQ-AS, the Body Shape Questionnaire and the author constructed items were calculated. The results of these analyses can be found in Table 1.

With respect to the participant responses on the Body Shape Questionnaire, the average score on this scale was a 76.73, with a median of 64 and standard deviation of 36.0. With respect to the MBSRQ-AS subscales, the mean score of the Appearance Evaluation subscale score was 3.69, with a median of 3.71 and standard deviation of 0.61. In comparison, the female population used to develop the MBSRQ scored an average of 3.36 on this scale (Cash, 2000). The mean score of the Appearance Orientation subscale was 3.61, with a median of 3.67 and standard deviation of .32 . Again, in comparison, the average score of women used in the scale development study was 3.91 (Cash, 2000). The Overweight Preoccupation scale average subscale is 2.49, with a median of 2.5 and a standard deviation of .99. Lastly, the Self-Classified Weight subscale yielded an average score of 3.29 , with a median of 3.0 and a standard deviation of .72. In comparison, the average score of women in the Cash study was 3.03 and 3.57, respectively, on the Overweight Preoccupation and Self-Classified Weight subscales. The average score on the Body Areas Satisfactions subscale was 3.62, with a median of 3.67 and a standard deviation of .57. The average score of women used in the scale development study was 3.91 (Cash, 2000). 
Specific attention was paid to numbers 28 and 29 of the MBSRQ-AS Body Area Satisfaction subscale, as they measure lower and mid-torso satisfaction, respectively. Descriptive statistics reveal a mean score of 3.48 and 3.04, and standard deviation of 1.19 and 1.43 for items 28 and 29, respectively. The median score of both of these items was four and three, respectively. As portrayed in Table 2, of the 75 individuals who completed item 28 , approximately $60 \%$ of these women were satisfied or very satisfied with their lower torso (buttocks, hips, thighs, legs), while $27 \%$ indicated being mostly or very dissatisfied. The remaining $13 \%$ indicated being neither satisfied nor dissatisfied with this area of their bodies. Seventy-six individuals completed number 29 of the Body Areas Satisfaction subscale, with $46 \%$ indicated satisfaction with their mid-torso (waist and stomach). Comparatively, $43 \%$ indicating being mostly or very dissatisfied with their waist and stomach, with $11 \%$ neither satisfied nor dissatisfied.

Finally, regarding participant responses on the author-constructed items that reflect how AA women feel about their bodies (see Table1), scores ranged from 1 (definitely disagree) to 5 (definitely agree). The mean score of author item 1 (I am satisfied with my body) was 3.45 , with a median of 4.0 and a standard deviation of 1.30 . The average score of author item 2 (I am satisfied with the flatness of my stomach) was 2.73, with a median of 2.0 and a standard deviation of 1.36). For author item 3 (I am satisfied with the narrowness of my waist), the mean score was 3.34, with a median of 4.0 and standard deviation of 1.37. The average score of author item 4 (As long as my stomach is flat, my weight doesn't matter) was 2.85 , with a median of 3.0 and standard deviation of 1.07. Lastly, the mean of author item 5 (As long as my waist is narrow, my weight doesn't matter) was 2.62 , with a median of 3.0 and standard deviation of 0.85 . As portrayed in Table 3, of the 71 individuals who completed these author constructed items, 
specific to item 1, $65 \%$ were satisfied with their bodies, while $28 \%$ reported some level of dissatisfaction and 7\% indicated being neither satisfied nor dissatisfied. In terms of author item 2, 28\% were satisfied with the flatness of their stomachs, while 53\% indicated dissatisfaction. As well, $15 \%$ indicated neither satisfaction nor dissatisfaction. Author item 3, which examined satisfaction with narrowness of waist, indicated that 53\% of respondents were indeed satisfied with their waist. Comparatively, $34 \%$ were dissatisfied and $8 \%$ were neither satisfied nor dissatisfied. In response to author item 4 , $33 \%$ agreed that as long as their stomach was flat, their weight did not matter. In contrast, $45 \%$ disagreed with this statement and $22 \%$ neither agreed nor disagreed. Lastly, 17\% agreed that as long as their waist was narrow, their weight did not matter; while $48 \%$ disagreed with this statement and 35\% neither agreed nor disagreed. Study Question \#2: What do AA college women say is the ideal body figure for AA women? What is the BMI of these shapes?

To determine what AA women report to be the ideal figure for AA, as well as the BMI of these shapes, responses on the Reese Figure Rating Scale (Patt, Lane, Finney, Yane, \& Becker, 2002) were examined. Regarding the Reese Figure Rating scale, 2\% of participants indicated their ideal size was figure three (BMI of $37.2 \pm 2$ ); $4 \%$ indicated figure five (BMI OF 35.4 \pm 8 ); $12 \%$ indicated figure six (BMI of 31.0 \pm 4 ); $30 \%$ indicated

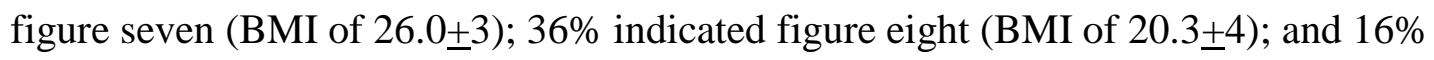

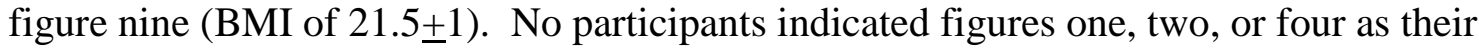
ideals. 
Study Question \#3: What is the level of discrepancy between the ideal and actual body shapes of AA women?

In order to determine the level of discrepancy between actual and ideal body shapes, both actual and ideal body shapes on the Reese Figure Rating Scale were examined. The average actual Reese Figure Shape chosen by participants was 6.5, with a standard deviation of 1.86. Comparatively, the average ideal body figure chosen was seven, with a standard deviation of 1.86 .

Regarding the Reese Figure Rating scale, $10 \%$ of participants indicated their current size was figure three (BMI of 37.2 \pm 2 ); recall from the analyses for the research question above that $2 \%$ indicated that this was their ideal size. Ten percent indicated figure four (BMI of 31.8+2) as their actual size, whereas (as indicated above), no participants indicated this as their ideal size. Nine percent indicated figure five (BMI of $35.4 \pm 8)$ as their actual size, again in comparison to $4 \%$ who indicated this as their ideal size. Nine percent indicated figure six (BMI of $31.0 \pm 4$ ) as their actual size, while $12 \%$ said this was their ideal size. In terms of Figure seven (BMI of 26.0+3), 31\% indicated this was their actual size, while a similar percentage (30\%) also indicated this was their ideal size. Finally, 17\% indicated figure eight (BMI of 20.3 $\underline{2}$ ) and $14 \%$ indicated figure nine (BMI of 21.5+1) as their actual sizes, as compared to $36 \%$ and $16 \%$ indicating these as their ideal size, respectively.

In order to determine the difference between actual and ideal body shapes, a discrepancy score was calculated for each participant who completed the Reese Figure Rating Scale. Discrepancy scores were calculated by subtracting the number corresponding to the participant's ideal image from the number corresponding with their reported actual size image. A discrepancy score was not calculated for individuals that 
did not indicate both their current and ideal body shapes. Given that higher rating scale numbers on the Reese correspond with smaller sizes, a positive discrepancy score means that individuals desire to be larger than they are and a negative discrepancy score indicated that individual desire to be smaller than they are. Thirty-eight individuals had a negative discrepancy score, whereas seven individuals had a positive discrepancy score. In other words, 38 individuals desired to be smaller and seven desired to be larger. Furthermore, twenty individuals had a discrepancy score of zero, indicating no difference between their actual and ideal body shapes. Of those who had a positive discrepancy score, all had a score of 1 , indicating that they desired to be one size bigger than their current size as portrayed on the Reese. Of those who had a negative discrepancy score, these scores ranged from -4 to -1 , with a mean of -1 and a SD of 0.98 . Across all participants, the average discrepancy score was a -1 , with a median of -1 and standard deviation of 1.37 .

Study Question \#4: Is AA college women's level of satisfaction with their mid and lower torso related to their overall satisfaction with their appearance and their level of concern regarding their body shape?

In order to determine how African American women's level of satisfaction with their mid and lower torso related to their, several correlation analyses were run. These analyses are presented in Tables 4 and 5. All correlations were significant. To illustrate, item 28, item 29 and the mean of these two items from the Body Area Satisfaction scale correlated, respectively, at .659, .658, and .740 with the Appearance Evaluation subscale of the MBSRQ. All correlations were significant at the $\mathrm{p}<.01$ level. This suggests that as satisfaction with mid and lower torso increase, satisfaction with appearance increased as well. Similarly, also significant at the .01 level, item 28, item 29 and the mean of 
these two items from the Body Area Satisfaction scale correlated, respectively, at -.624, .751 , and -.780 with scores on the Body Shape Questionnaire. Specifically, BSQ correlations with items twenty-eight and twenty-nine indicate a negative relationship. Therefore, as individuals' satisfaction with their mid and lower torso increased, their concern with (i.e., preoccupation with) their body shape decreased. As well, the correlation with the average of these items indicates that when both of these items are combined, they have a strong negative correlation with the Body Shape Questionnaire. Similar patterns were found for the correlations between the author constructed items assessing satisfaction with the flatness of one's stomach and the narrowness of one's waist were found, again reflecting the importance of these body parts for overall body satisfaction among this AA female college sample.

Study Question \#5: Is AA women's satisfaction with their mid and low torso related to the discrepancy they report between their actual and ideal shape?

Correlation analyses were also run to determine how satisfaction with mid and lower torso related to the discrepancy score on the Reese Figure Rating Scale (Patt, Lane, Finney, Yane, \& Becker, 2002), which was previously calculated by subtracting the individual's ideal from actual body shape indicated on this rating form. As can be seen in Tables 6 , the correlation analysis between the discrepancy score and item twenty-eight on the Body Areas Satisfaction scale was not statistically significant. This suggests that there is no correlation between the difference between participants' actual and ideal selves and their satisfaction with their mid-torso. On the other hand, the correlations between the discrepancy score and item twenty-nine (-.39), as well as the correlation between the discrepancy score and the average of items twenty-eight and twenty-nine (.36) are statistically significant at the .05 level. These negative correlations suggests that 
as an individuals' satisfaction with their lower torso and overall satisfaction with mid and lower torso increase, the difference between their real and ideal selves may decrease slightly. Further, as depicted in Table 7, correlation analysis indicated that there is a significant and negative relationship between the discrepancy score and the second and third author constructed items (-.49 and -.40 , respectively). These analyses suggest that as participants' satisfaction with their waist and stomach increased, the difference between their real and ideal selves decreased as well.

Study Question \#6: Does AA college women's satisfaction with mid and lower torso mediate the relationship between BMI (weight) and overall satisfaction with appearance?

The mediation model was tested using the procedures outlined by Baron and Kenny (1986). Baron and Kenny state that the following criteria must be met for mediation to be possible: (1) the independent variable (BMI) must be significantly related to the mediator (satisfaction with mid and lower torso); (2) the independent variable (BMI) must be significantly related to the dependent variable (satisfaction with appearance); (3) the mediator (satisfaction with mid and lower torso) must be significantly related to the dependent variable (satisfaction with appearance); and (4) the relationship between the independent variable (BMI) and the dependent variable (satisfaction with appearance) must be reduced when the mediator (satisfaction with mid and lower torso) is included in the regression equation.

Four sets of regressions were conducted that tested the hypothesis that the relationship between BMI and overall satisfaction with appearance is mediated by satisfaction with mid and lower torso. After running these regressions, standardized regression weights Beta's were compared to determine the degree to which the mediator 
(satisfaction with mid and lower torso) accounted for the independent variable (BMI) effects on satisfaction with appearance.

Analyses indicated a full mediation between mid and lower torso and the relationship between BMI (weight) and satisfaction with appearance. First, the average of items twenty-eight and twenty-nine of the MBSRQ-AS Body Area Satisfaction Subscale were regressed onto BMI, with results indicating a significant and negative relationship $([$ beta $]=-.551)$; therefore as BMI increases, satisfaction with mid and lower torso decreases. Second, by regressing MBRSQ-AS Appearance Evaluation subscale scores onto BMI, results indicate a significant and negative relationship ([beta] = -.516); therefore, as BMI increases, satisfaction with appearance decreases. Third, Appearance Evaluation scores were regressed onto the average of items twenty-eight and twenty-nine of the MBSRQ-AS Body Areas Satisfaction subscale, which measure satisfaction with mid and lower torso, respectively. This analysis indicated a significant and positive relationship $([$ beta $]=.740)$ in which as satisfaction with mid and lower torso increased, satisfaction with appearance increased as well. Lastly, when regressing Appearance Evaluation scores onto BMI and the average of twenty-eight and twenty-nine of the MBSRQ-AS, the beta coefficient for BMI was no longer significant (-.140), while the coefficient for the average of twenty-eight and twenty-nine remained significant (.682). This suggests that the relationship between satisfaction with appearance and BMI is mediated by satisfaction with mid and lower torso.

Study Question \#7: Does AA college women's satisfaction with mid and lower torso mediate the relationship between their BMI and their level of concern regarding body shape? 
Four sets of regressions were conducted that tested the hypothesis that the relationship between BMI and level of concern with body shape is mediated by satisfaction with mid and lower torso. After running these regressions, standardized regression weights Beta's were compared to determine the degree to which the mediator (satisfaction with mid and lower torso) accounted for the independent variable (BMI) effects on the dependent variable (body shape concerns).

These analyses indicated a partial mediation between mid and lower torso and the relationship between BMI and body shape concerns. First, the previously reported regression of the average of 28 and 29 of the MBSRQ-AS Body Area Satisfaction subscale onto BMI continues to have a negative and significant relationship ([beta] = .551). Second, by regressing Body Shape Questionnaire (BSQ) scores onto BMI, results indicate a significant and positive relationship ([beta] $=.660)$, indicating that as BMI increased, concerns with body shape also increased. Third, BSQ scores were regressed onto the average of items twenty-eight and twenty-nine on the Body Areas Satisfaction subscale, which measure satisfaction with mid and lower torso, respectively. This analysis indicated a significant and negative relationship ([beta] $=-.780)$, in which, as satisfaction with mid and lower torso increased, concern with body shape decreased. Lastly, when regressing BSQ scores onto BMI and the average of items twenty-eight and twenty-nine, the relationship between BMI and BSQ scores is significant ([beta] = .324) while the relationship between BMI and the two items from the Body Areas Satisfaction subscale was also significant $([$ beta $]=-.609)$. Partial mediation was indicated due to the fact that when regressing BSQ scores onto BMI and the average of items twenty-eight and twenty-nine, the relationships between BMI and body shape and BMI and the two items from the Body Areas Satisfaction subscale were both significant. This indicates 
that the relationship between BMI and body shape concerns is accounted for, in part, by satisfaction with mid and lower torso.

Study Question \#8: Does African American college women's satisfaction with their stomach and waist, as measured by the author constructed items, mediate the relationship between their BMI and their overall satisfaction with appearance?

Four sets of regressions were conducted that tested the hypothesis that the relationship between BMI and overall satisfaction with appearance is mediated by satisfaction with flatness of stomach and narrowness of waist, as measured by items two and three of the author constructed scale. After running these regressions, standardized regression weights Beta's were compared to determine the degree to which the mediator (satisfaction with flatness of stomach and narrowness of waist) accounted for the independent variable (BMI) effects on the dependent variable (overall satisfaction with appearance).

These analyses indicated a full mediation between satisfaction with stomach and waist and the relationship between BMI and overall satisfaction with appearance. First, the average of author items two and three were regressed onto BMI, resulting in a negative and significant relationship $([$ beta $]=-.687$. Second, the previously reported regression of Appearance Evaluation scores of the MBSRQ-AS onto BMI continues to be significant $([$ beta] $=-.516)$. Third, Appearance Evaluation scores were regressed onto the average of the author constructed items that measured satisfaction with stomach and waist, indicating a significant and positive relationship ([beta] $=.678)$. This indicates that as satisfaction with stomach and waist increased, satisfaction with appearance also increased. Finally, Appearance Evaluation scores were regressed onto both BMI and the two author constructed items, resulting in the relationship between Appearance 
Evaluation Scores and BMI becoming insignificant ([beta] $=-.105)$ while the relationship between Appearance Evaluation Scores and the author constructed items remained significant $([$ beta $]=.598)$, revealing a full mediation relationship. Specifically, the relationship between overall satisfaction with appearance and BMI is accounted for by satisfaction with waist and stomach.

Study Question \#9: Does African American college women's satisfaction with their stomach and waist, as measured by the author constructed items, mediate the relationship between their BMI and their level of concern with body shape?

Finally, four sets of regressions were conducted that tested the hypothesis that the relationship between BMI and level of concern with body shape is mediated by satisfaction with mid and lower torso. After running these regressions, standardized regression weights Beta's were compared to determine the degree to which the mediator (satisfaction with mid and lower torso) accounted for the independent variable (BMI) effects on the dependent variable (level of concern with body shape).

These analyses indicated a full mediation between satisfaction with stomach and waist and the relationship between BMI and level of concern with body shape. First, the average of author constructed items two and three were regressed onto BMI, and continued to result in a negative and significant relationship ([beta] $=-.687$. Second, by regressing the Body Shape Questionnaire (BSQ) scores onto BMI, significance, as indicated earlier, was present $([$ beta $]=.660)$. Third, the BSQ scores were regressed onto the average of the author constructed items that measure stomach and waist satisfaction, resulting in a negative and significant relationship ([beta] $=-.788$ ). This indicates that as satisfaction with waist and stomach increased, body shape concerns decreased. Finally, the BSQ scores were then regressed onto BMI and satisfaction with stomach and waist. 
Specifically, the relationship between BMI and BSQ scores was no longer significant $([$ beta $]=.230)$, yet the relationship between the author constructed items and BSQ scores remained significant $([$ beta $]=-.626)$. These findings indicate that the relationship between BMI and body shape concerns can be accounted for by satisfaction with waist and stomach.

\section{Exploratory Analyses}

Responses on the author constructed item free-response question were reviewed, examined, and categorized by body area (i.e. breasts, thighs, stomach, buttocks, etc.). Fifty-nine participants responded to the free-response question, "If anything, what would you change about your body shape?" Twelve percent of participants indicated they would make their breasts smaller, while $10 \%$ indicated they would increase the size of their breasts. Nineteen percent of respondents indicated the desire for smaller or more toned thighs, while $17 \%$ wanted larger buttocks. Furthermore, $41 \%$ of respondents indicated wanting a flatter stomach and $15 \%$ wanted a smaller waist. Lastly, $5 \%$ of participants indicated that they wanted to gain weight and $12 \%$ reported being satisfied with their shape and/or curves and would not change anything. 


\section{Chapter Five: Discussion}

The purpose of this study was to gain more insight into how African American women feel about their body shape. Due to the contradictory results of previous research, the current study aimed to more adequately and appropriately measure the level of satisfaction African American women have with their body image, and in particular, specific areas of their body. This chapter discusses the results of this study, as presented in the previous chapter. First, the findings are summarized and subsequently discussed in reference to the previous literature; such discussion is presented by study questions. Next, limitations of the study are reviewed. Finally, suggestions are made for future research, and implications for counseling interventions are discussed.

Summary of Results and Relationship to Previous Literature

Study Question \#1: What do African American (AA) college women report regarding their level of satisfaction with their bodies and shape?

How AA women in this study felt about their bodies was gathered through three inventories: The Body Shape Questionnaire (BSQ; Cooper, Taylor, Cooper, and Fairburn, 1987), the Multidimensional Body-Self Relations Questionnaire-Appearance Scales (MBSRQ-AS; Cash, 2000), and author constructed questions.

Responses on the BSQ are provided on a six point Likert-type scale ranging from (1) never to (6) always. Thus, possible scores range from 34 - 204, with high scores indicating more concern and lower scores indicating less concern with body shape. Specific to this study's population, the average score on the BSQ was 76.73, suggesting that participants had some level of concern with their body shape.

In terms of the MBSRQ-AS, which measures overall satisfaction with appearance, the participants in this study had a mean score of 3.69 on the Appearance 
Evaluation Scale, a scale which ranges from (1) strongly disagree to (5) strongly agree. This mean score suggests that the current sample of AA college women had positive feelings toward and were satisfied with their appearance. Additionally, this mean score was slightly higher than the average score (3.36) of the female population used to develop the scale which was comprised mainly of Caucasian women (Cash, 2000). Although comparative data was not obtained in this study, for theoretical reasons noted in the literature review, this finding nevertheless works in concert with those of several researchers who have concluded that African American women tend to be more satisfied with their appearance than other women. Specifically, past researchers have found that Black women have scored higher on the Appearance Evaluation subscale of the MBSRQAS as compared to women of other races (e.g., Miller, Gleaves, Hirsch, Green, Snow, and Corbett, 2000; Snooks and Hall, 2002), supporting the idea that AA women are more satisfied with their appearance than other races of women (Cash, Morrow, Hrabosky, and Perry, 2004).

In contrast, the overall score on the Appearance Orientation subscale of the MBSRQ-AS, which measures cognitive and behavioral investment in one's appearance, was a 3.61 for the women in this study (subscale scores range from (1) strongly disagree to (5) strongly agree). This score was slightly lower than the average score of the original scale construction population (3.91; Cash, 2000). This finding suggests that the women in the current student were less preoccupied with their appearance than the instrumentation's norm sample group. Comparatively, this finding does not support Cash, Morrow, Hrabosky, and Perry's (2004) study in which Black women were actually more invested in their appearance than their non-Black counterparts. 
The Overweight Preoccupation scale also has scores which range from (1) strongly disagree to (5) strongly agree; this scale assesses fat anxiety, weight vigilance, dieting and eating restraint. The Self-Classified Weight scale assess self and other perceptions of weight by having respondents choose responses ranging from (1) very underweight to (5) very overweight. In reference to the Overweight Preoccupation and Self-Classified Weight subscale, scores (2.49 and 3.29, respectively) were lower than the sample population used to create the MBSRQ (3.03 and 3.57, respectively; Cash, 2000). These findings again suggest that the current study's participants were less anxious about being fat and less vigilant about their weight than the previous norm group, which included a majority of Caucasian women. As well, the participants in this study reported believing that they and others would classify themselves as normal to somewhat overweight.

Particular attention was paid to the Body Areas Satisfaction subscale due to the fact that this subscale includes items that assess satisfaction with specific areas of the body, rather than overall body satisfaction The average subscale score (3.62) was lower than that of the original norm population (3.23, Cash, 2000). This finding suggests that the current study's participants experienced slightly lower satisfaction with their bodies, than did the original population. Further attention was paid to items 28 and 29 of this subscale, which measure satisfaction with lower and mid torso, respectively. This special attention was paid to these items due to research findings that suggest that Black women may pay particular attention to the waist, hips, and buttocks, when assessing body satisfaction (Falconer \& Neville, 2000; Befort, Thomas, Daley, Rhode, and Ahluwalia, 2008). Overall, the results indicated that the majority of participants were, on some level, satisfied with their mid and lower torso. Specifically, 58\% were satisfied with their lower 
torso and $44 \%$ were satisfied with their mid torso. Furthermore, $15 \%$ and $14 \%$ were neither satisfied nor dissatisfied (i.e., felt neutral about) with these areas. Taken together, this means that $27 \%$ and $42 \%$, respectively, were dissatisfied with the mid and lower torso. These findings support the previous result of African American women being satisfied with their bodies (Demarest \& Allen, 2000), yet it also lends further support to the idea that overall satisfaction does not translate to satisfaction with all areas of the body (Befort, et al., 2008) and suggests that feelings about specific body areas, such as the waist and stomach, may be important in determining African American women's body satisfaction.

Information gathered from the author constructed items further bolsters this finding. Specifically, responses to these items suggest that the majority of participants were not satisfied with the flatness of their stomachs, despite over half being satisfied with the narrowness of their waist. Furthermore, $32 \%$ and $17 \%$, respectively, agreed that flatness of stomach or narrowness of waist were more important than actual weight. This finding seems to suggest that the women in this study were concerned with both their body shape and their overall weight.

In summary, it appears that the women in this study were satisfied with their appearance. Yet, despite this overall satisfaction, it is evident that the participants were dissatisfied with specific areas of their bodies, mainly their mid and lower torsos. Therefore, this study not only captured the level of overall satisfaction, but it also provided information regarding the body area dissatisfaction that African American women may experience.

Study Question \#2: What do AA college women say is the ideal body figure for AA women? What is the BMI of these shapes? 
Although the average size of the participants in this study was smaller than the original Reese Figure Scale study participants, both groups indicated a similar ideal. Specifically, the average size of participants in this study was 6.86 , which falls between a body mass index range of $26.0 \pm 3$ and $31.0 \pm 4$. On the other hand, the ideal size indicated by participants was 7.50 , which falls within a body mass index range of $20.3 \pm 2$ and 26.0 \pm 3. Interestingly, according to the authors of the Reese Figure Rating Scale, most participants were slightly overweight to obese and had an ideal that falls within the normal to slightly overweight categories. Furthermore, although the original study's population and the current study's population differed in terms of actual body weight and size, both samples had similar desires in terms of what they believed to be the most attractive body shape for themselves, which again was normal to slightly overweight according to BMI standards.

Study Question \#3: What is the level of discrepancy between the ideal and actual body shapes of AA women?

Most participants in this study wanted to be at least one size smaller than their current body size. Specifically, rather than having a body mass index of $26.0 \pm 3$, they desired to have a body mass index of $20.3 \pm 2$. Based on the criteria from the developers of the Reese Figure Rating Scale, as well as from standard BMI interpretations, most participants were slightly overweight, with a desire to be of normal weight (Patt, Lane, Finney, Yane, \& Becker, 2002). These findings support the idea that African American women do indeed have some level of body dissatisfaction. Furthermore, the Reese Figure Rating Scale was developed by increasing the BMI of each image, paying particular attention to thighs, abdomen and breasts. This suggests that the participants 
may have wanted to decrease these specific areas in order to be more satisfied with their body shape.

Study Question \#4: Is AA women's level of satisfaction with their mid and lower torso related to their overall satisfaction with appearance and their level of concern regarding their body shape?

The correlation analyses reveal that as participants' satisfaction with their mid and lower torso increased, their overall satisfaction with appearance did as well. The analyses of the responses to the author constructed items revealed similar correlations. Therefore, when examining these results, it can be said that decreases in the size of the abdomen, waist and thighs (areas of the mid and lower torso), lead African American women to be more satisfied with their overall physical attractiveness. Furthermore, correlations analyses revealed that as participants' satisfaction with their mid and lower torso increased, their concern with their body shape, as measured by the Body Shape Questionnaire, decreased. Certainly, these results lend credence to the notion that for AA women, these particular body parts are a key to overall satisfaction with one's appearance.

Study Question \#5: Is AA women's satisfaction with their mid and low torso related to the discrepancy they report between their actual and ideal shape?

Individuals' overall satisfaction with their mid and lower torso, as measured by item 28, item 29 of the MBSRQ-AS, and the author constructed items measuring waist and stomach satisfaction, was significantly related to the difference between their actual and ideal selves. Specifically, as participants' satisfaction with these specific body areas increased, the discrepancy between their actual and ideal selves decreased. Therefore, the 
more content the women in this study were with their mid and lower torso, the closer to their own ideal shape and size that they were.

Study Question \#6: Does AA Women's satisfaction with their mid and lower torso mediate the relationship between BMI (weight) and overall satisfaction with appearance?

These regression analyses support the previous results that indicate a significant relationship between BMI and overall satisfaction with appearance. They also suggest a relationship between body mass index and mid and lower torso satisfaction. As body mass index increased, overall satisfaction with appearance and satisfaction with mid and lower torso decreased. It may be that a higher body mass index indicated an increase in size of the specific areas of the mid and lower torso, resulting in less satisfaction with overall appearance. Indeed, as satisfaction with mid and lower torso increased, so did satisfaction with overall appearance. Therefore, it appears that body mass index and overall satisfaction with appearance are related (i.e., as weight goes up, satisfaction goes down), and that satisfaction with mid and lower torso influences this relationship. Therefore a full mediation relationship was revealed suggesting that those individuals who were satisfied with their mid and lower torso were most satisfied with their overall appearance.

Study Question \#7: Does AA college women's satisfaction with mid and lower torso mediate the relationship between their BMI and their level of concern regarding body shape?

Results of these analyses continue to lend support to the idea that body satisfaction and level of concern regarding body shape are affected by both body mass index and mid and lower torso satisfaction. As previously reported, higher body mass index was related to dissatisfaction with mid and lower torso. As well, as body mass 
increased, concern with body shape also increased. Specific to body shape, as an individual's satisfaction with mid and lower torso increased, their concern with body shape decreased. It can thus be said that overall concern with body shape is influenced by both body mass index and mid and lower torso satisfaction. Therefore, as an individual's body size increased, her body shape concerns increased as well. Yet, the results of the analyses suggest that satisfaction with mid and lower torso influences the relationship between body size and body shape concern. Consequently a partial mediation relationship indicated that those individuals who were satisfied with their mid and lower torso and had less concern with their body shape.

Study Question \#8: Does African American college women's satisfaction with their stomach and waist, as measured by the author constructed items, mediate the relationship between their BMI and their overall satisfaction with appearance?

As satisfaction with narrowness of waist and flatness of stomach increased, body mass index decreased. In other words, participant's perceptions that their stomachs were flat, and their waist narrow, was related to a lower overall weight, As well, satisfaction with waist and stomach resulted in satisfaction with overall appearance. This indicates that women with narrower waists and flatter stomachs were possibly at a lower weight than those dissatisfied with their bodies. Continuing, satisfaction with these areas also contributed to a positive evaluation of appearance, yet appearance evaluation had no significant relationship with body mass index. Overall, these analyses indicate that satisfaction with waist and stomach mediates, or influences, the relationship between body mass index and appearance evaluation. Consequently, satisfaction with waist and stomach positively influenced appearance evaluation, despite body mass index. In 
essence, then, for these Black college women, it was satisfaction with stomach and waist that was key to appearance satisfaction, not weight per se.

Study Question \#9: Does African American college women's satisfaction with their stomach and waist, as measured by the author constructed items, mediate the relationship between their BMI and their level of concern regarding their body shape?

As satisfaction with waist and stomach increased, concern for body shape decreased. Yet, as body mass index increased, concern for body shape increased as well. Therefore, the relationship between body mass index and body shape concern can be accounted for by the level of satisfaction with waist and stomach. This finding, which reflects a full mediation relationship, demonstrates that satisfaction with waist and stomach leads to less body shape concern, despite overall body mass index.

\section{Exploratory Examinations}

Author Constructed Item Free Response. Despite the overall satisfaction previously reported by study participants, there were also indications of these participants wanting to change specific areas of their bodies. Most of the women who responded to the free response question wanted to make their breasts smaller, reduce the size of their thighs, have a flatter stomach, have a narrower waist, and have larger buttocks. At the same time, many women indicated they would not change anything about their bodies and wanted to maintain their shape/curves. This finding supports Falconer and Neville's (2000) assumption that the African American culture values a curvy body with a larger buttocks and hips. It may be that the women in the current study were satisfied with their bodies overall, but had less satisfaction in specific areas of their shapes.

Overall Summary of Findings 
Taken together, the findings reflect a group of AA women who, in terms of body mass index, were considered to be between overweight and obese. Related to this, participants wanted to reduce their body size to categories that fell within the normal to slightly overweight range. These women were satisfied with their overall appearance, but had some dissatisfaction with their mid and lower torsos. As well, participants paid close attention to the flatness of their stomachs and the narrowness of their waists. Therefore, despite being satisfied with bodies that are considered beyond a normal weight range, participants were not satisfied with all areas of their bodies. These findings lend support to the idea that African American women's actual satisfaction with their bodies may be more adequately and thoroughly understood by not only measuring overall satisfaction, but also assessing satisfaction with specific areas.

\section{Limitations}

Although great care was taken to ensure a strong methodology, as with any research study, there are still a number of limitations. Still, prior to discussing these limitations, it is first important to note the strengths of this study.

A first strength of this study was the focus on African American women. Unlike previous research, this study focused on a completely African American sample, without comparing them to other races that may hold different body image ideals. Much of past body satisfaction research has excluded African American women, purportedly due to a societal myth that women of color do not experience distressed body image (Yankowski, 2000). Furthermore, when studies do include African American women, they are often compared to White women (Miller, Gleaves, Hirsch, Green, Snow, and Corbett, 2000) and other women of color (Snooks and Hall, 2002), making it difficult to understand the individual experiences of African American women. By focusing on an African 
American population, and using a figure rating scale developed for and based on Black women (i.e. Reese Figure Rating Scale; Patt, Lane, Finney, Yane, \& Becker, 2002), this study allows the ability to pay particular attention to this group.

Another strength of this study is that it is only the second study in existence to gather information on AA women's satisfaction with specific body areas, rather than to examine global satisfaction only, which may obscure more specific results. As previously reported, very limited research exists that examines satisfaction with specific body areas. Rather, the vast majority of previous research has only reported overall body satisfaction of African American women (Demarest \& Allen, 2000; Lokken, Worthy, Ferraro, \& Attman, 2008; Patel \& Gray, 2001). The current study adds to the limited research on specific body area satisfaction, (Befort, Thomas, Daley, Rhode, and Ahluwalia, 2008), and lends support to the idea that satisfaction with specific body areas may influence body image satisfaction of African American women.

Despite the major strengths described above, limitations, of course, exist. First, despite being specific to African American women, the generalizablity of results may be limited due to the homogeneity of the participants. All participants were undergraduate students at a large Midwestern university, and these AA students may differ from other African American students across the country. As well, it may be that graduate students or women that fall outside of the age range of the current population may have different experiences with their level of body satisfaction. Additionally, no assessment of acculturation to the White or Black cultures was given. Instruments that measured these constructs may have provided additional results that could possibly account for within group differences. Despite this, inclusion would have increased the length of the online survey, likely decreasing response rate. Additionally, the inclusion of such an instrument 
would have followed previous research's tendency to compare African American women to the dominant White culture, and thus was purposefully not done. This study should be replicated with African American women that may vary in age and level of education, and perhaps also in terms of level of acculturation, as this would allow for a greater ability to generalize to different women within the African American population.

Second, the methodology was solely comprised of an online survey that required participants to self-report their height/weight, as well as determine their actual and ideal sizes from a visual representation of the Reese Figure Rating Scale on a computer screen. Due to the importance of understanding the relationship of body mass index to several instruments and constructs within the study, it may have been more prudent to use a methodology in which the researcher measured the height and weight of each participant, rather than relied on self-report. Moreover, some individuals may have had a difficult time determining their actual and ideal figures on a computer screen, suggesting that it may have been useful to have the instruments in a paper-and-pencil format that would have allowed for a possibly clearer picture of the images with which participants were presented.

Third, the sample population size was smaller than comparable quantitative studies that examined body image within the African American community (comparatively, Falconer and Neville's 2004 study had a sample size of 124). This smaller sample size may also affect the generalizablity of findings. It may be that the limits placed on the desired population (i.e., African American, female, undergraduate) made it difficult to recruit a significant number of participants. Also, the previously discussed methodology (online survey) may not have been a method that some individuals were comfortable with. Still, it may be that some individuals were not 
interested in participating in a research study due to the negative historical relationship between the African American/Black population and scientific research. Research shows that individuals within the African American population are historically difficult to recruit due to issues of suspicion and distrust (Freimuth, Quinn, Thomas, Cole, Zook, and Duncan, 2001). In addition, despite the current author being a Black woman, Freimuth et al. (2001) suggest that this alone may not be enough to increase a feeling of trust between investigator and participant.

\section{Implications for Future Research}

Several modifications to the existing study are possible, some of which have been mentioned throughout the previous section. The following section will discuss further ways to add to the research literature in this area.

First, although this study was quantitative in nature and included instruments that were normed on African American populations, it may be beneficial to replicate this study with different instruments designed to measure the dependent variables. If similar findings were obtained across diverse instruments, this would certainly bolster one's faith in the accuracy of the findings. Also, given the information gathered from the free response question of the author constructed items, it seems important to conduct additional qualitative studies on AA women's body shape satisfaction. As was the case with this study, Befort et al. (2001) also uncovered information regarding African American women's satisfaction with certain areas of their bodies. Both studies found that satisfactions with the waist and stomach were important to AA women. This suggests a possibility that widely used measures (with only a few items assessing satisfaction with these body parts) may not be adequately representative of the experiences of African American women. A future study could take a qualitative or more mixed methods 
approach to gather more information regarding shape satisfaction among AA women. This would also allow for the opportunity to discuss factors that contribute to how these women determine their ideal shape, and subsequently, what leads them to both be satisfied overall, yet dissatisfied with specific areas. Some research has suggested that African American women tend to value skin color (Falconer \& Neville, 2000), hair, confidence, overall appearance (Befort, Thomas, Daley, Rhode, \& Ahluwalia, 2008), and style of dress (Rubin, Fitts, \& Becker, 2003) in determining their satisfaction with their appearance. Likewise, while this study focused mainly on the lower and upper torso as important to Black women's body shape satisfaction, the results of the free responses indicate that a study focusing on the size and shape of the buttocks, could also shed additional light on the important topic of how AA women view their bodies. By employing a qualitative method, future research may be able to determine how all of these factors (e.g., satisfaction with torso areas, buttocks, skin color, dress) are weighted in determining appearance satisfaction. An eventual goal would be to create a body satisfaction measure specific to, and normed with AA women, that captures their lived and culture-specific experiences.

Second, as previously reported, this study included only undergraduate women at a predominantly white institution. Future studies could focus on individuals from universities across the country, including at historically black colleges and universities, in order to provide for the ability to generalize to additional AA populations. Furthermore, research that includes graduate and professional students would allow for a greater cross section of this population. Furthermore, it may be important to begin developing research studies and programs that are geared toward girls and young women. This focus would allow for the opportunity to validate and monitor how younger women feel about 
their shapes and bodies. Finally, accessing community members that are not a part of the academic community would allow for a richer set of data that would span across the various socio-economic statuses. By having a more inclusive sample population, the ability to explore possible factors that affect shape and body area satisfaction would increase.

Third, future research should continue to be attuned to the best methods of data collection and participation within the African American community. Care and understanding should be employed in assisting African American participants to feel more at ease and aware of the research process, implications, and individual/cultural benefits. For example, providing verbal explanation of the purpose of the research with each participant, or possibly through community resources (such as churches) that hold specific functions for African Americans may be beneficial. Furthermore, encouraging leaders within the community to act as liaisons between researchers and participants, and agreeing to provide research results and implications to participants or chosen community leaders, may also increase the level of interest and participation. These activities may allow for a more positive relationship of trust to develop between scientists and this specific racial/ethnic minority group.

\section{Implications for Prevention and Therapy}

Although it is important for researchers and those in the helping profession to avoid making premature judgments based on the results of this study, there are several implications for outreach and therapy that can be ascertained. First, given that both research and practice have often assumed that AA women do not experience body concerns, these results can assist in increasing awareness of the concerns that AA women do experience regarding their body satisfaction and shapes. Indeed, current prevention 
programming geared toward White women and other women of color should be considerate of the African American experience and make modifications where necessary. Specifically, avoiding solely focusing on a thin ideal, it may be appropriate to also include information regarding body shape ideals that are more applicable to African American women.

Being that body dissatisfaction can lead to significant psychological distress, dieting behaviors, negative affect and eating disorders, including self-starvation, bingeeating and purging (Thompson \& Stice, 2001; Williamson, 1998), it is prudent that the African American female population be provided with resources and programs that cater to their specific needs and concerns. Specifically, knowledge of the differences in ideals and ways of determining body satisfaction for African American women can assist in constructing programs that are aimed at maintaining a healthy and desired weight and shape. Specifically, since this study revealed that many of the participants are slightly overweight, education on proper nutrition and exercise would be beneficial. Providing information on how to maintain a healthy and appropriate body weight would possibly allow for even more satisfaction with body shape.

In reference to therapy, awareness that disorders related to body image dissatisfaction are not color-blind can greatly influence practice within the helping professions. It is important for counselors to be aware of the possible cultural context within which African American women view their bodies. Counselors should not only assess for body image satisfaction, but they should also assess for shape and specific area contentment. This would allow for a more comprehensive picture of how African American female clients view themselves related to their bodies.

\section{Conclusion}


The current study is one of the first to examine body area satisfaction within the African American community. As well, by focusing solely on the African American community, rather than comparing across racial/ethnic minority groups, this study allows for an acknowledgement and validation of African American women and their individual experiences. The findings of this study provide several significant contributions to existing literature. First, results demonstrated that African American college women are satisfied with their overall appearance and body shape. Second, despite overall satisfaction, African American college women indicated dissatisfaction with certain areas of their bodies, specifically the mid and lower torso. In short, then, although the results indicate that study participants were satisfied with their bodies, it also lends support to the idea that they are not completely satisfied with all areas of their bodies. This suggests that it is of the utmost importance that research, similar to the current study, continues to examine this phenomenon. Additional research should continue to explore how overall satisfaction among AA women may be part-specific. For AA women, where body satisfaction is concerned, the parts appear to be greater than the whole. It is hoped that this study will serve as a starting point for new and exciting research in the area of body shape within the African American female community and will contribute to the important work of validating the experiences of Black women. 


\section{REFERENCES}

Anderson, L.A., Janes, G.R., Ziemer, D.C., Phillips, L.C. (1997). Diabetes in urban African Americans. Body image, satisfaction with size, and weight change attempts. The Diabetes Educator, 23(30, 301-308.

Ard, J.A., Greene, L.F., Malpede, C.Z., \& Jefferson, W.K. (2007). Association between body image disparity and culturally specific factors that affect weight in Black and White women. Ethnicity \& Disease, 17(2), S2-34-S2-39.

Baldwin, J. A,, \& Bell, Y. R. (1985). The African Self-Consciousness Scale: An Africentric personality questionnaire. Western Journal of Black Studies, 9, 61-68.

Baron, R.M., \& Kenny, D.A. (1986). The moderator-mediator variable distinction in social psychological research: conceptual, strategic, and statistical considerations. Journal of Personality and Social Psychology, 51, 1173-1182.

Befort, C.A., Thomas, J.L., Daley, C.M., Rhode, P.C., \& Ahluwalia, J.S. (2006). Perceptions and beliefs about body size, weight, and weight loss among obese African American women: A qualitative study. Health Education \& Behavior, $35(3), 410-426$.

Borzekowski, D.L.G., Robinson, T.N., \& Killen, J.D. (2000). Does the camera add 10 pounds? Media use, perceived importance of appearance, and weight concerns among teenage girls. Journal of Adolescent Health, 26(1), 36-41.

Botta, R.A. (2000). The mirror of television: A comparison of Black and White adolescents' body image. Journal of Communications, 50(3)144-159.

Bracken, B.A. (1992). Multidimensional self concept scale. Austin, TX: Pro-Ed. 
Cash, T. F. (2000). MBSRQ User's Manual (3rd ed.). Unpublished manuscript.

Cash, T.F., Morrow, J.A., Hrabosky, J.I., \& Perry, A.A. (2004). How has body image changed? A cross-sectional investigation of college women and men from 19832001. Journal of Consulting and Clinical Psychology, 72(6), 1081-1089.

Cooper, P.J., Taylor, M.J., Cooper, Z., \& Fairburn, C.G. (1987). The development and validation of the body shape questionnaire. International Journal of Eating Disorders, 6(4), 485-494.

Demarest, J., \& Allen, R. (2000). Body image: Gender, ethnic, and age differences. The Journal of Social Psychology, 140(4), 465-472.

Falconer, J.W., \& Neville, H.A. (2000). African American college women's body image: An examination of body mass, African self-consciousness, and skin color satisfaction. Psychology of Women Quarterly, 24(3), 236-243.

Franzoi, S., \& Shields, S. (1984) . The body self-esteem scale: Multidimensional structure and sex differences in a college population. Journal of Personality Assessment, 48, 173-178.

Freimuth, V.S., Quinn, S.C., Thomas, S.B, Cole, G., Zook, E., \& Duncan, T. (2001). African Americans' views on research and the Tuskegee Syphilis study. Social Science and Medicine, 52(5), 797-808.

Garner, D.M. (1991). Eating disorder inventory-2. Odessa, FL: Psychological Assessment Resources, Inc.

Garner, D. M., Olmstead, M. P., \& Polivy, J. (1983). Development and validation of a multidimensional Eating Disorder Inventory for anorexia nervosa and bulimia. International Journal of Eating Disorders, 2, 15-34.

Garner, D. M., \& Garfinkel, P. E. (1979). The Eating Attitudes Test: An index of the 
symptoms of anorexia nervosa. Psychological Medicine, 9, 273-279.

Gluck, M.E., \& Geliebter, A. (2002). Racial/ethnic differences in body image and eating behaviors. Eating Behaviors, 3(2), 143-151.

Gordon, K.H., Perez, M., \& Joiner, Jr., T.E. (2002). The impact of racial stereotypes on eating disorder recognition. International Journal of Eating Disorders, 32(2), 219-224.

Harris, S.M. (1995). Family, self, and sociocultural contributions to body-image attitudes of African-American women. Psychology of Women Quarterly, 19(1), 129-145.

Hrabosky, J.I., \& Grilo, C.M. (2007). Body image and eating disordered behavior in a community sample of Black and Hispanic women. Eating Behaviors, 8(1), 106114.

James, K.A., Phelps, L., \& Bross, A.L. (2001). Body dissatisfaction, drive for thinness, and self-esteem in African American college females. Psychology in the Schools, 38(6), 491-496.

Lokken, K.L., Worthy, S.L., Ferraro, F.R., Attmann, J. (2008). Bulimic symptoms and body image dissatisfaction in college women: More affected by climate or race? The Journal of Psychology, 14(2), 386-394.

Lynch, E., Liu, K., Spring, B., Hankinson, A., Wei, G.S., \& Greenland, P. (2007). The coronary artery risk development in young adults (CARDIA) study. American Journal of Epidemiology, 165(9), 1055-1062.

Marais, D.L., Wassenaar, D.R., \& Kramers, A.L. (2003). Acculturation and eating disorder symptomatology in Black men and women. Eating and Weight Disorders, 8(1), 44-54.

Miller, K.J., Gleaves, D.H., Hirsch, T.G., Green, B.A., Snow, A.C., \& Corbett, C.C. 
(2000). Comparisons of body image dimensions by race/ethnicity and gender in a university population. International Journal of Eating Disorders, 27(3), 310-316.

Patel, K.A., \& Gray, J.J. (2001). Judgement accuracy in body preference among African Americans. Sex Roles, 44(3-4), 227-235.

Patt, M.R., Lane, A.E., Finney, C.P., Yankey, L.R., \& Becker, D.M. (2002). Body image assessment: Comparison of figure rating scales among urban Black women. Ethnicity \& Disease, 12(1), 54-62.

Perez, M., \& Joiner, Jr., T.E. (2003). Body image dissatisfaction and disordered eating in Black and White women. International Journal of Eating Disorders, 33(3), 342350.

Poran, M.A. (2006). The politics of protection: Body image, social pressures, and the misrepresentation of young Black women. Sex Roles, 55(11-12), 739-755.

Rosen, J.C., Jones, A., Ramirez, E., \& Waxman, S. (1996). Body shape questionnaire: Studies of Validity and Reliability. International Journal of Eating Disorders, 20(3), 315-319.

Rubin, L.R., Fitts, M.K., \& Becker, A.E. (2003). Whatever feels good in my soul: Body ethics and aesthetics among African American and Latina women. Culture, Medicine, and Psychiatry, 27, 49-75.

Schooler, D., Ward, L.M., Merriwether, A., \& Caruthers, A. (2004). Who's that girl: Television's role in the body image development of young White and Black women. Psychology of Women Quarterly, 28(1), 38-47.

Silberstein, L., Striegel-Moore, R., Timko, C., \& Rodin, J. (1988) . Behavioral and psychological implications of body dissatisfaction: Do women and men differ? Sex Roles, 19, 219-232. 
Snooks, M.K, \& Hill, S.K. (2002). Relationship of body size, body image, and selfesteem in African American, European American, and Mexican American middle class women. Health Care for Women International, 23, 460-466.

Striegel-Moore, R.H., Schrieber, G.B., Pike, K.M., Wilfley, D.E., \& Rodin, J. (1995). Drive for thinness in Black and White preadolescent girls. International Journal of Eating Disorders, 18(1), 59-69.

Stunkard, A. J., Sorensen, T., \& Schulsinger, F. (1983). Use of the Danish adoption register for the study of obesity and thinness. In S. Kety, L. P. Rowland, R. L. Sidman, \& S.W. Matthysse (Eds.), The genetics of neurological and psychiatric disorders (pp. 115-120). New York: Raven Press.

Thomas, V.G. (2001). Body-image satisfaction among Black women. The Journal of Social Psychology, 129(1), 107-112.

Thompson, J.K., \& Stice, E. (2001). Thin-ideal internalization: Mounting evidence for a new risk factor for body-image disturbance and eating pathology. Current Directions in Psychological Science, 181-183.

Webb, T.T., Looby, E.J., \& Fults-McMurtery, R. (2004). African American men’s perceptions of body figure attractiveness: An acculturation study. Journal of Black Studies, 34(3), 370-385.

White, M.A., \& Grilo, C.M. (2005). Ethnic differences in the prediction of eating and body image disturbances among female adolescent psychiatric inpatients. International Journal of Eating Disorders, 38(1), 78-84.

White, M.A., Kohlmaier, J.R., Varnado-Sullivan, \& Williamson, D.A. (2003). 
Racial/ethnic differences in weight concers: Protective and risk factors for the development of eating disorders and obesity among adolescent females. Eating and Weight Disorders, 8(1), 20-25.

Williamson, D.A. (1990). Assessment of eating disorders: Obesity, anorexia, and bulimia. New York, NY: Pergamon Press.

Yanovski, S.Z. (2000). Eating disorders, race, and mythology. Archives of Family Medicine, 9, 88. 


\section{Appendix A \\ Multidimensional Body Self-Relations Questionnaire-Appearance Scales}

Please indicate your level of agreement with each of the following statements by circling the number that corresponds most closely with your own feeling.

$\begin{array}{lllll}1 & & & \\ \text { Strongly } & \text { Moderately } & \text { Neither } & \text { Moderately } & \text { Strongly } \\ \text { Disagree } & \text { Disagree } & \text { Agree } & \text { Agree } & \text { Agree } \\ & & \text { Nor } & & \\ & & \text { Disagree } & & \end{array}$

\begin{tabular}{|c|c|c|c|c|c|}
\hline 1. Before going public, I always notice how I look. ${ }^{\text {B }}$ & 1 & 2 & 3 & 4 & 5 \\
\hline 2. I am careful to buy clothes that will make me look my best. ${ }^{\mathrm{B}}$ & 1 & 2 & 3 & 4 & 5 \\
\hline 3. My body is sexually appealing. A & 1 & 2 & 3 & 4 & 5 \\
\hline 4. I constantly worry about being or becoming fat. ${ }^{\mathrm{C}}$ & 1 & 2 & 3 & 4 & 5 \\
\hline 5. I like my looks just the way they are. ${ }^{A}$ & 1 & 2 & 3 & 4 & 5 \\
\hline 6. I check my appearance in a mirror whenever I can. ${ }^{B}$ & 1 & 2 & 3 & 4 & 5 \\
\hline 7. Before going out, I usually spend a lot of time getting ready. ${ }^{\text {B }}$ & 1 & 2 & 3 & 4 & 5 \\
\hline 8. I am very conscious of even small changes in my weight. ${ }^{\mathrm{C}}$ & 1 & 2 & 3 & 4 & 5 \\
\hline 9. It is important that I always look good. ${ }^{\text {A }}$ & 1 & 2 & 3 & 4 & 5 \\
\hline 10. I use very few grooming products. ${ }^{B}$ & 1 & 2 & 3 & 4 & 5 \\
\hline 11. I like the way I look without my clothes on. ${ }^{B}$ & 1 & 2 & 3 & 4 & 5 \\
\hline 12. I am self-conscious if my grooming isn't right. ${ }^{A}$ & 1 & 2 & 3 & 4 & 5 \\
\hline $\begin{array}{l}\text { 13. Magazine articles are not an important source of information about fashion and } \\
\text { "being attractive." B }\end{array}$ & 1 & 2 & 3 & 4 & 5 \\
\hline 14. I usually wear whatever is handy without caring how it looks. ${ }^{B}$ & 1 & 2 & 3 & 4 & 5 \\
\hline 15. I like the way my clothes fit me. ${ }^{A}$ & 1 & 2 & 3 & 4 & 5 \\
\hline 16. I don't care what people think of my appearance. ${ }^{\text {B }}$ & 1 & 2 & 3 & 4 & 5 \\
\hline
\end{tabular}



17. I take special care of my hair grooming.
18. I dislike my physique.

$\begin{array}{lllll}1 & 2 & 3 & 4 & 5\end{array}$
19. I am physically unattractive. $\begin{array}{lllll}1 & 2 & 3 & 4 & 5\end{array}$
20. I never think about my appearance. ${ }^{\text {B }}$
21. I am always trying to improve my physical appearance. ${ }^{\mathrm{B}}$

$\begin{array}{lllll}1 & 2 & 3 & 4 & 5\end{array}$
22. I am on a weight-loss diet. ${ }^{\mathrm{C}}$

$\begin{array}{lllll}1 & 2 & 3 & 4 & 5\end{array}$
23. I have tried to lose weight by fasting or going on crash diets.
23. I have tried to lose weight by fasting or going on crash diets. ${ }^{\mathrm{C}}$
a. Never
b. Rarely
c. Sometimes
d. Often
e. Very often

24. I think I am: ${ }^{\mathrm{E}}$
f. Very underweight
g. Somewhat underweight
h. Normal weight
i. Somewhat overweight
j. Very overweight

25. From looking at me, most other people think I am: ${ }^{\mathrm{E}}$
a. Very underweight
b. Somewhat underweight
c. Normal weight
d. Somewhat overweight
e. Very overweight

Use the scale to indicate how dissatisfied or satisfied you are with each of the following areas or aspects of your body.

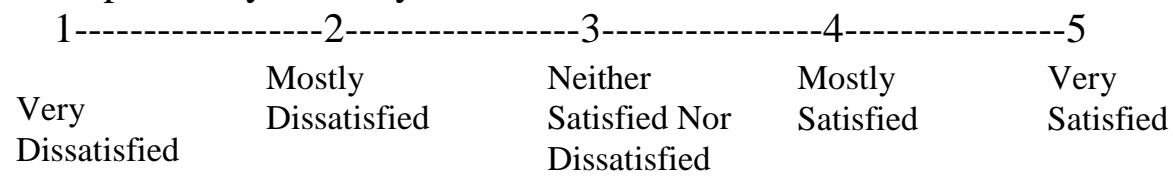

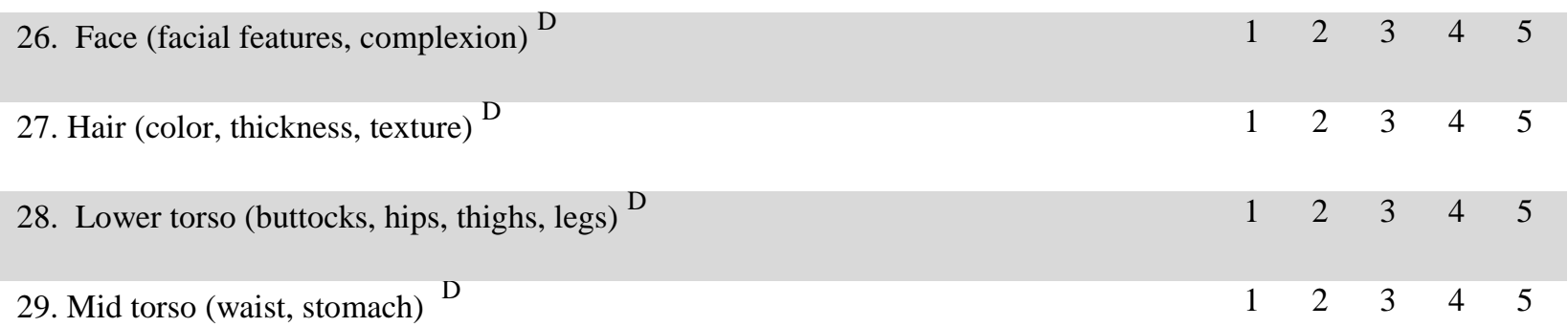




\begin{tabular}{|c|c|c|c|c|c|}
\hline 30. Upper torso (chest or breasts, shoulders, arms) & 1 & 2 & 3 & 4 & 5 \\
\hline 31. Muscle tone ${ }^{\mathrm{D}}$ & 1 & 2 & 3 & 4 & 5 \\
\hline 32. Weight ${ }^{\mathrm{D}}$ & 1 & 2 & 3 & 4 & 5 \\
\hline 33. Height $\mathrm{D}$ & 1 & 2 & 3 & 4 & 5 \\
\hline 34. Overall appearance ${ }^{D}$ & 1 & 2 & 3 & 4 & 5 \\
\hline
\end{tabular}

Subscales as follows: $\mathrm{A}=\mathrm{Appearance}$ Evaluation; $\mathrm{B}=$ Appearance Orientation; $\mathrm{C}=$ Overweight Preoccupation; $\mathrm{D}=$ Body Areas Satisfaction; E=Self-Classified Weight 


\section{Appendix B \\ Body Shape Questionnaire}

We should like to know how you have been feeling about your appearance over the PAST FOUR WEEKS. Please read each question and circle the appropriate number to the right. Please answer all the questions.

OVER THE PAST FOUR WEEKS:

1. Has feeling bored made you brood about your shape?

\begin{tabular}{|c|c|c|c|c|c|}
\hline Never & Rarely & Sometimes & Often & $\begin{array}{l}\text { Very } \\
\text { Often }\end{array}$ & Always \\
\hline 1 & 2 & 3 & 4 & 5 & 6 \\
\hline
\end{tabular}

2. Have you been so worried about your shape that you have been feeling that you ought to diet?

1 2 3

$4 \quad 5$

3. Have you thought that your thighs, hips or bottom are too large for the rest of you?

1

2

3

$\begin{array}{lll}4 & 5 & 6\end{array}$

4. Have you been afraid that you might become fat (or fatter)?

5. Have you worried about your flesh not being firm enough ?

6. Has feeling full (e.g., after eating a large meal) made you feel fat?

8. Have you avoided running because your flesh might wobble?

9. Has being with thin women made you feel self-conscious about your shape?

10. Have you worried about your thighs spreading out when sitting down?

12. Have you noticed the shape of other women and felt that your own shape compared unfavourably? 
Never Rarely Sometimes Often Often Always

13. Has thinking about your shape interfered with your ability to concentrate (e.g., while watching television, reading, listening to conversations)?

14. Has being naked, such as when taking a bath, made you feel fat?

3

4

$5 \quad 6$

15. Have you avoided wearing clothes which make you particularly aware of the shape of your body?

2

3
4

$5 \quad 6$

16. Have you imagined cutting off fleshy areas of your body?

17. Has eating sweets, cakes, or other high calorie food made you feel fat?

2

3

$4 \quad 5$

18. 18. Have you not gone out to social occasions (e.g., parties) because you have felt bad about your shape?

19. Have you felt excessively large and rounded?

20. Have you felt ashamed of your body?

3

$\begin{array}{lll}4 & 5 & 6\end{array}$

21. Has worry about your shape made you diet?

22. Have you felt happiest about your shape when your stomach has been empty (e.g., in the morning)?

23. Have you thought that you are the shape you are because you lack self-control?

24. Have you worried about other people seeing rolls of flesh around your waist or stomach?

25. Have you felt that it is not fair that other women are thinner than you? 
Never Rarely Sometimes Often Often Always

26. Have you vomited in order to feel thinner?

1

2

3

45

6

27. When in company have you worried about taking up too much room (e.g., sitting on a sofa or a bus seat)?

1

2

3

4

56

28. Have you worried about your flesh being dimply?

2

3

$4 \quad 5$

6

29. Has seeing your reflection (e.g., in a mirror or shop window) made you feel bad about your shape?

1

2

3

$\begin{array}{lll}4 & 5 & 6\end{array}$

30. Have you pinched areas of your body to see how much fat there is?

1

2

3

4

5

6

31. Have you avoided situations where people could see your body (e.g., communal changing rooms or swimming baths)?

32. Have you taken laxatives in order to feel thinner?

33. Have you been particularly self-conscious about your shape when in the company of other people?

34. Has worry about your shape made you feel you ought to exercise? 
Appendix C

Reese Figure Rating Scale

Which figure best fits where you are now?:

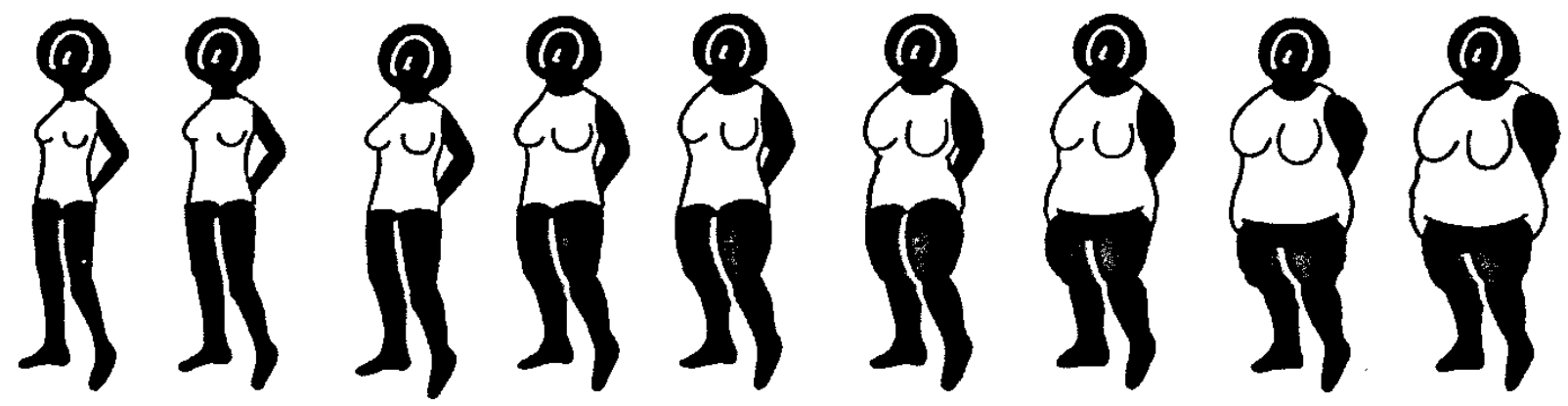

$\begin{array}{lllllllll}9 & 8 & 7 & 6 & 5 & 4 & 3 & 2 & 1\end{array}$

Mean BMI $\left(\mathrm{kg} / \mathrm{m}^{2}\right)$ of each image:

$\begin{array}{lllllllll}21.5 \pm 1 & 20.3 \pm 2 & 26.0 \pm 3 & 31.0 \pm 4 & 35.4 \pm 8 & 31.8 \pm 2 & 37.2 \pm 10 & 42.0 \pm 4 & 44.3 \pm 4\end{array}$

Which figure would be ideal for you now?:

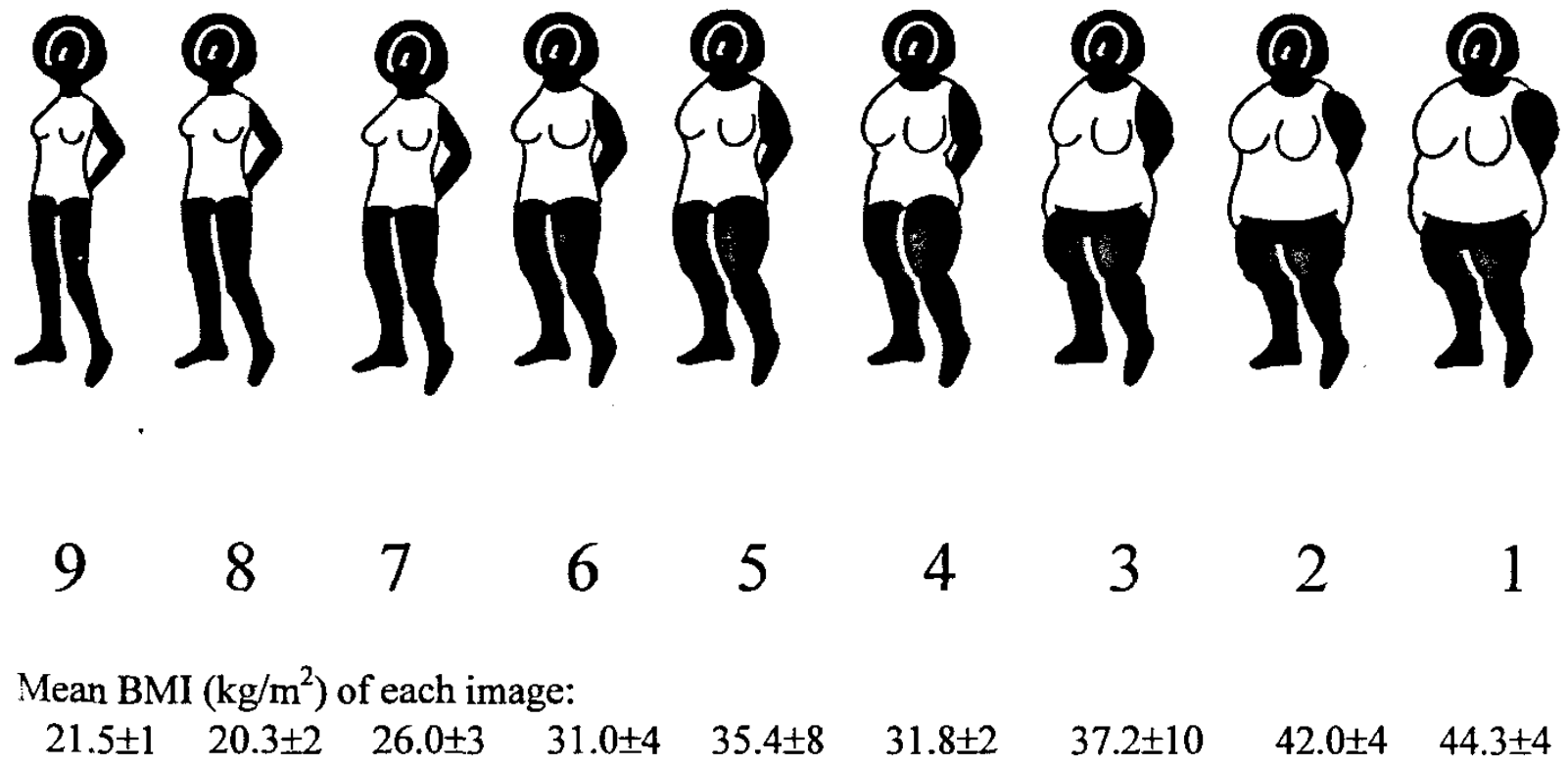


Below are the original questions used to create the Reese Figure Rating Scale. Only questions 1 and 3 will be used in the current study.

1. Which figure best fits where you are now?

2. Which figure is most like what you were when you were 21 ?

3. Which figure would be ideal for you now?

4. Which figure do you think you will be like a year from now?

5. Which figure is most like the majority of other women you know who are your age?

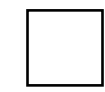

6. Which figure is likely to be the healthiest?

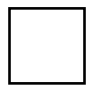

7. Which figure do you think is ideal for women over 40 ? 


\section{Appendix D \\ Author Constructed Items}

Please indicate your level of agreement with each of the following statements by circling the number that corresponds most closely with your own feeling.

1. I am satisfied with my body.

$\begin{array}{ccccc}1 & 2 & 3 & 4 & 5 \\ \text { Definitely } & & \text { Neutral } & & \begin{array}{c}\text { Definitely } \\ \text { Agree }\end{array}\end{array}$

2. I am satisfied with the flatness of my stomach.

$\begin{array}{llccc}1 & 2 & 3 & 4 & 5 \\ \text { Definitely } & & \text { Neutral } & & \text { Definitely } \\ \text { Disagree } & & & & \text { Agree }\end{array}$

3. I am satisfied with the narrowness of my waist.

$\begin{array}{llccc}1 & 2 & 3 & 4 & 5 \\ \text { Definitely } & & \text { Neutral } & & \text { Definitely } \\ \text { Disagree } & & & & \text { Agree }\end{array}$

4. As long as my stomach is flat, my weight does not matter.

$\begin{array}{llccc}1 & 2 & 3 & 4 & 5 \\ \text { Definitely } & & \text { Neutral } & & \text { Definitely } \\ \text { Disagree } & & & & \text { Agree }\end{array}$

5. As long as my waist is narrow, my weight does not matter.

$\begin{array}{llccc}1 & 2 & 3 & 4 & 5 \\ \text { Definitely } & & \text { Neutral } & & \text { Definitely } \\ \text { Disagree } & & & & \text { Agree }\end{array}$

6. If anything, what would you change about your body shape? 


\section{Appendix E Demographic Questionnaire}

Please read the items below and answer to the best of your knowledge.

1. Age:

2. Height:

3. Weight:

4. Which of the following best describes your race/ethnicity?
a. African-American/Black
b. Hispanic-American/Latino/Chicano
c. Native-American/American Indian
d. Asian-American/Pacific Islander
e. Caucasian/European American/White
f. Multiracial
g. Other

5. What is your current relationship status?
a. Single, no partner
b. Dating casually
c. Dating seriously
d. In a married or committed relationship
e. Divorced
f. Other

6. Sex
a. Male
b. Female
c. Transgendered

7. Sexual Orientation
a. Gay or lesbian
b. Bisexual
c. Transgender
d. Transsexual
e. Heterosexual 
8. Religion
a. Judaism
b. Christianity
c. Muslim
d. Buddhist
e. Hindu
f. Chinese Folk
g. Tribal Religions
h. New Religions
i. Non-religious
j. Atheist
k. Agnostic
1. Other

9. Approximate income level:

If self-supporting, please report own income. If supported by parent(s) or guardian(s) please report that income:
a. Less than $\$ 15,000$
b. $\$ 15,000-\$ 25,000$
c. $\$ 25,000-\$ 50,000$
d. $\$ 50,000-\$ 75,000$
e. $\$ 75,000-\$ 100,000$
f. $\$ 100,000$ and above

10. What do you consider your socioeconomic status to be?
a. Working class
b. Middle class
c. Upper middle class
d. Upper class
e. Other

11. Your class standing can be best described as:
a. Freshman
b. Sophomore
c. Junior
d. Senior
e. Graduate student
f. Other 
12. Are you an international student?
a. Yes
b. No

13. Residence
a. Residence Hall/Dormitory
b. Fraternity/Sorority
c. On-Campus apartment
d. Off-Campus apartment/house
e. With partner/spouse
f. With parents
g. Other

14. What is your college cumulative GPA? 


\section{Appendix F}

MU Info Ad

\section{Seeking African American Undergraduate Women for a Dissertation Research Study}

Seeking African American/Black undergraduate women who are interested in contributing information to learn more about the ideal body shape of Black women. This study is part of a dissertation research study on body image among African American/Black college women conducted by Rashanta A. Bledman. The purpose of this research study is to gain more information regarding how African American/Black women perceive their bodies. Study participants will be asked to fill out several online questionnaires related to how they, as Black women, feel about their shape. The study is completely confidential. To enroll, email Rashanta A. Bledman at rabwd2@mail.mizzou.edu.

Announcement sponsored by Laurie Mintz, Doctoral Advisor, ESCP Department 


\section{Appendix G \\ Email Notification}

Dear Student,

I am a graduate student in the Department of Educational, School, and Counseling Psychology conducting a study on body image among African American/Black college women. Individuals eligible for this study include female undergraduate students who identify as Black or African American. I am emailing to ask you to participate in this study that I am conducting which will, hopefully, help us to better understand how Black women feel about their bodies. Your participation will not only help us to gain more insight into how Black/African American women view their bodies, but it will also help psychologists interested in working with the Black/African American community. This study is web-based and will take approximately 30 minutes to complete. You will first be asked to answer several demographic questions. Next, you will be provided with several surveys to complete. Following the completion of the study, you will be provided with information regarding the purpose of the research and information on how to contact the researcher or others for assistance with concerns about body-image or any other psychological issues. All information you provide in this study is anonymous, and I will have no way to identify you or associate your answers with you. Finally, after you complete the study, you will be taken to a website to enter into a drawing for one of two $\$ 50$ gift certificates to the University of Missouri Bookstore. If you would like to participate, please click on the link provided below. If you have any questions for me prior to participating in the study, you may contact me, Rashanta A. Bledman, M.Ed., at bledmanra@missouri.edu. 


\section{Appendix $\mathbf{H}$ \\ Email to various Black/African American organizations}

I am currently a 5th yr doctoral student in Counseling Psychology in the process of completing my dissertation entitled The Ideal Body Shape of African American/Black college women. I have done several discussion groups on and off campus over the past years at MU discussing body image of Black women; which significantly influenced my dissertation topic. I would like to reach out to more of the undergraduate community, specifically Black women, and wondered if I would be able to do this through (Organization). There is not enough information on how Black women feel about their bodies, and I am trying to expand the research to include our perspective. I am wondering if members of (Organization) would be willing to receive an email regarding voluntarily participating in my study. I would be more than willing to talk to the officers and/or members about the study and provide more information.

I appreciate your taking the time to read this email and look forward to your response. Also, if you feel that my request is not feasible, I would greatly appreciate any referral to other possible avenues.

Thank you,

Rashanta A. Bledman, M.Ed.

Doctoral Candidate

Department of Educational, School, and Counseling Psychology

University of Missouri

Columbia, MO 65202

rabwd2@mail.mizzou.edu 
Appendix I

Recruitment Flyer

\section{Ideal Body Shape Dissertation Study}

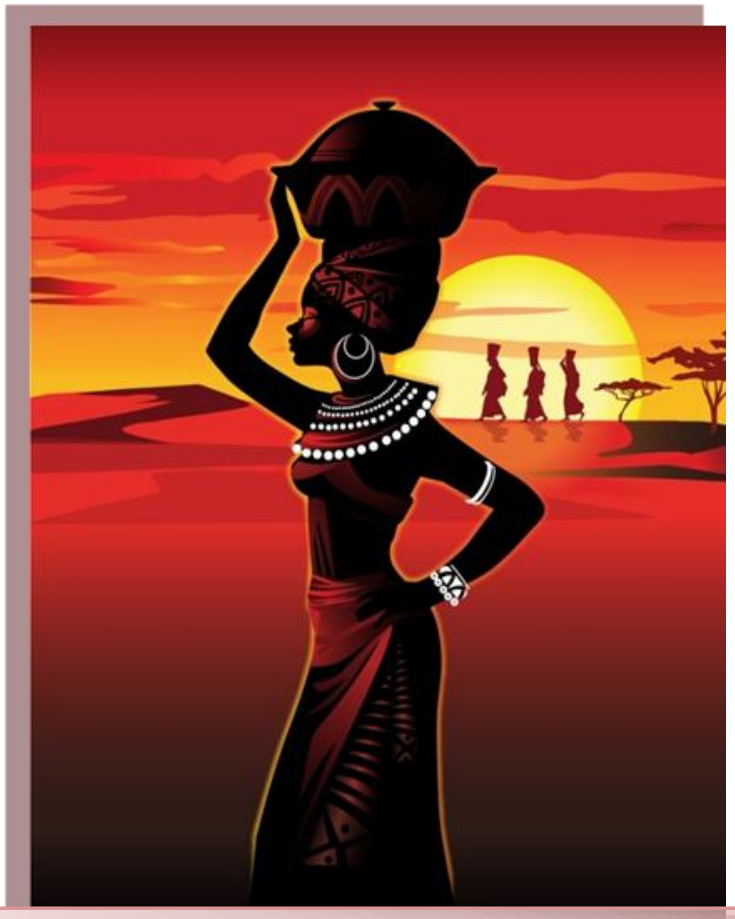

Are you an African

American/Black

undergraduate

woman?

Are you interested in learning more about the ideal body shape of African American/Black women?

- If you answered YES to these questions, you may be eligible to participate in a dissertation study on body shape and African American/Black women

- The purpose of this research study is to gain more information regarding how African American/Black women perceive their bodies. Participation in the study may have the benefit of making participants more aware of their body image and ideal shape.

- Adults (age 18+) are eligible to participate.

For more information, please contact Rashanta A. Bledman at rabwd2@mail.mizzou.edu

\begin{tabular}{|c|c|c|c|c|c|c|c|c|c|}
\hline 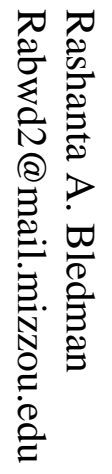 & 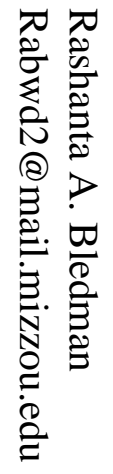 & 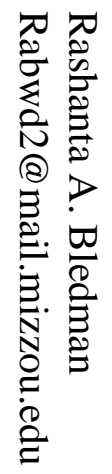 & 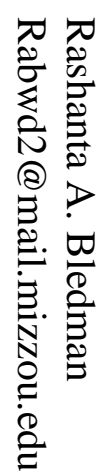 & 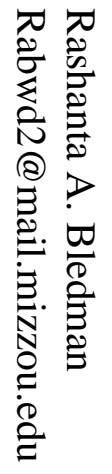 & 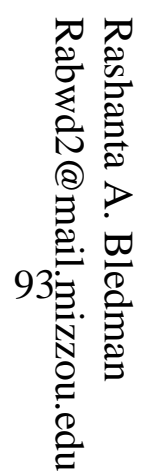 & 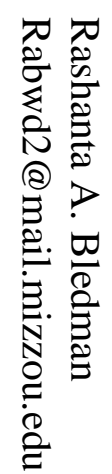 & 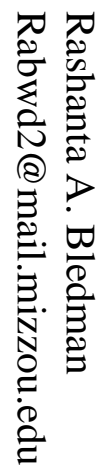 & 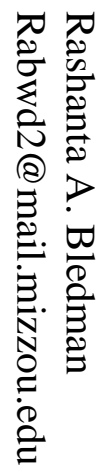 & 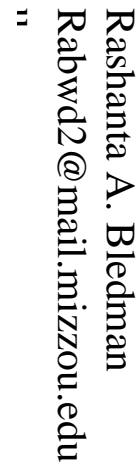 \\
\hline
\end{tabular}




\section{Appendix J \\ Informed Consent}

\section{Purpose and Expected Benefits}

You are invited to participate in a study on body image among African American/Black college women conducted by Rashanta A. Bledman, M.Ed, under the supervision of Laurie Mintz, Ph.D. The purpose of this study is to gain more information regarding how African American/Black women perceive their bodies.

\section{Participant Responsibilities}

You will be asked to read and click on 'agree with terms of informed consent' giving your consent to participate in this study. By agreeing to the terms of this consent form, you understand that your participation will involve the following: a) completing a demographic questionnaire and b) completing several questionnaires regarding your feelings toward your body shape. These questionnaires should take you 30 minutes to complete.

\section{Possible Risks and Benefits}

While this study poses no greater risk than those ordinarily experienced in everyday life, many of the questions in this study relate to personal attitudes and beliefs regarding body shape and size, some of which may be sensitive or may make you feel uncomfortable. Please understand that your completion of this study is complete voluntary and that there is no penalty for refusal to participate. Also, please understand that you may stop completion of this project at any time if you feel bothered with the content of the activities, and may refuse to answer a question(s) if you so choose. Participation in the study may also have the benefit of making you more aware of your body image and those of other African American women.

\section{Compensation}

After completion of the study, you will be entered into a drawing for one of two $\$ 50$ gift certificate to the MU bookstore. After all data is collected, two winners will be randomly drawn and notified via email. Also, if you are randomly selected to receive a questionnaire again in two weeks, you will have the opportunity to enter into an additional raffle for one $\$ 50$ gift certificate to the MU bookstore. However, please realize that you may only enter the prize raffle after you complete the entire study.

\section{Confidentiality}

The information that you contribute is private and anonymous. No one outside the researcher will have access to the completed data. Although internet transmission of data is not completely secure and thus, complete confidentiality cannot be assured, no identifying information will be asked for during any portion of the study and once data is downloaded, the website will be dismantled. Results of this study may be published in 
scientific journals, but you will not be identified in any such publication. Also, the results of this research will be made available to you upon request.

You will be asked for your MU pawprint at the beginning of the survey. Your pawprint will only be used to enter you into the raffle (described above), to contact you if you are a winner, and for the follow-up study (i..e, to randomly select those for the study and to contact those chosen). After your participation and the raffle are complete, the pawprint will be removed and no longer connected to your survey responses. Please do not put your name or other identifying information on the surveys.

If you have any questions about this study, you may contact Rashanta A. Bledman (email: bledmanra@missouri.edu). Also, you may direct any questions about the use of human subjects in research to the Campus Institutional Review Board Office at the University of Missouri at the phone number (573) 882-9585, fax number (573) 884-0663, or e-mail address umcresearchcirb@missouri.edu.

Completion of the survey indicates you have read the information above and any questions that you have asked have been answered to your satisfaction. A copy of this form is available to you upon request.

Your participation in this study is greatly appreciated. Thank you for your time. 


\section{Appendix K \\ Debriefing Form}

Thank you very much for your participation in this study. The purpose of this study is to gain more information regarding how African American/Black women perceive their bodies and shapes.

Previous research on body image has concentrated primarily on European American women. Research that has included African American/Black women has been inconclusive, with some results indicating that African American/Black women are satisfied with their bodies, and others indicating dissatisfaction. Despite these discrepancies, little research has further evaluated how African American/Black women feel about their bodies and shapes.

This study may help to answer the question of how African American/Black college women view their bodies, and especially, their shapes. As well, it may lend insight into whether or not African American/Black women place more emphasis on certain areas of their bodies in order to determine overall body shape satisfaction.

If you are concerned about your body image or shape (or any other issue), the University Counseling Center offers free counseling to students. You may contact them for an appointment by calling 882-6601.

If you have any questions/comments about the procedures or this study, you may contact Rashanta A. Bledman at bledmanra@missouri.edu. 


\section{Appendix L \\ Test-Retest Email}

\section{Dear Student,}

As I hope you recall, two weeks ago you completed surveys online for a study of body image among African American/Black college women. You have been randomly selected to complete a follow-up questionnaire. Your selection had nothing to do with your answers on the first survey; you were randomly selected. I am hoping you will assist me by completing one additional questionnaire, which should take you approximately 10 minutes to complete.

At the end of completion, you will be taken to a website to enter into a drawing for one $\$ 50$ gift certificate to the University of Missouri bookstore.

If you are willing to participate, please click on the link below.

If you have any questions for me prior to participating in the study, you may contact me, Rashanta A. Bledman, M.Ed., at rabwd2@mail.mizzou.edu. 


\section{Appendix M \\ Test-Retest Debriefing Form}

Thank you very much for your participation in the follow-up survey for this study. The purpose of this study is to gain more information regarding how African American/Black women perceive their bodies and shapes.

Previous research on body image has concentrated primarily on European American women. Research that has included African American/Black women has been inconclusive, with some results indicating that African American/Black women are satisfied with their bodies, and others indicating dissatisfaction. Despite these discrepancies, little research has further evaluated how African American/Black women feel about their bodies and shapes.

This study may help to answer the question of how African American/Black college women view their bodies, and especially, their shapes. As well, it may lend insight into whether or not African American/Black women place more emphasis on certain areas of their bodies in order to determine overall body shape satisfaction.

If you are concerned about your body image or shape (or any other issue), the University Counseling Center offers free counseling to students. You may contact them for an appointment by calling 882-6601.

If you have any questions/comments about the procedures or this study, you may contact Rashanta A. Bledman at bledmanra@missouri.edu. 


\title{
Table 1
}

Means, Medians, Standard Deviations for Study Scales

\begin{tabular}{lccc} 
Variable & Mean & Median & SD \\
\hline BSQ (74) & 76.73 & 64.0 & 36.0
\end{tabular}

MBSRQ-AS (79)

$\begin{array}{lccc}\text { Appearance Evaluation } & 3.69 & 3.71 & 0.61 \\ \text { Appearance Orientation } & 3.61 & 3.67 & 0.32 \\ \text { Body Areas Satisfaction } & 3.62 & 3.67 & 0.57 \\ \text { Overweight Preoccupation } & 2.49 & 2.5 & 0.99 \\ \text { Self-Classified Weight } & 3.29 & 3.0 & 0.72 \\ \text { Author1 (71) } & 3.45 & 4.0 & 1.30 \\ \text { Author2 (71) } & 2.73 & 2.0 & 1.36 \\ \text { Author3 (71) } & 3.34 & 4.0 & 1.37 \\ \text { Author4 (71) } & 2.85 & 3.0 & 1.07 \\ \text { Author5 (71) } & 2.62 & 3.0 & 0.85\end{array}$

Note. The number in parentheses is the number of participants who completed this scale or item. BSQ=Body Shape Questionnaire. MBSRQ-AS=Multidimensional Body-Self Relations QuestionnaireAppearance Scales. Author1=I am satisfied with my body; Author2=I am satisfied with the flatness of my stomach, Author3=I am satisfied with the narrowness of my waist; Author4=As long as my stomach is flat, my weight does not matter; Author5=as long as my waist is narrow, my weight does not matter.

\begin{abstract}
Scores on the Body Shape Questionnaire can range from 34 - 204, with high scores indicating more concern and lower scores indicating less concern with body shape. Scores on the Appearance Evaluation Subscale can range from 1-5, with high scores indicating positive feelings and satisfaction with appearance, while lower scores indicate unhappiness with appearance. Scores on the Appearance Orientation Subscale can range from 1-5, with high scores indicating that an individual places significant importance on how they look, pays attention to their appearance, and engages in extensive self-grooming behaviors and lower scores are related to exhibit apathy toward appearance little importance placed on physical appearance. Scores on the Body Areas Satisfaction Subscale can range from. Scores on the Self-Classified Weight Subscale can range from. Scores on Author1 can range from 1 to 5, with high scores indicating a higher level of body satisfaction. Scores on Author2 can range from 1 to 5, with high scores indicating a higher level of satisfaction with flatness of stomach. Scores on Author3 can range from 1 to 5, with high scores indicating a higher level of satisfaction with narrowness of waist. Scores on Author 4 can range from 1 to 5, with high scores indicating a higher level of agreement with the idea that flatness of stomach is more important than weight. Scores on Author5 can range from 1 to 5, with high scores indicating a higher level of agreement with the idea that narrowness of waist is more important than actual weight.
\end{abstract}




\section{Table 2}

Number and Percentage of Responses for Items 28 and 29 of the Body Areas Satisfaction subscale

\begin{tabular}{lccccc} 
& 1 & 2 & 3 & 4 & 5 \\
Variable & $\begin{array}{c}\text { Very } \\
\text { Dissatisfied }\end{array}$ & $\begin{array}{c}\text { Mostly } \\
\text { Dissatisfied }\end{array}$ & $\begin{array}{c}\text { Neither Satisfied } \\
\text { Nor Dissatisfied }\end{array}$ & $\begin{array}{c}\text { Mostly } \\
\text { Satisfied }\end{array}$ & $\begin{array}{c}\text { Very } \\
\text { Satisfied }\end{array}$ \\
\hline Item 28 & $4(5)$ & $17(21.5)$ & $12(15)$ & $29(37)$ & $17(21.5)$ \\
Item 29 & $15(19)$ & $18(23)$ & $11(14)$ & $19(24)$ & $16(20)$
\end{tabular}

Note. $\mathrm{N}=79$. The number in parenthesis is the percent of respondents indicating this response). Item 28, from the Body Areas Satisfaction subscale of the MBSRQ-AS, measures satisfaction with lower torso (butt, hips, thighs, legs); Item 29, from the Body Areas Satisfaction subscale of the MBSRQ-AS, measures satisfaction with mid torso (waist and stomach). 


\section{Table 3}

Number and Percentage of Responses for Author Constructed Items

\begin{tabular}{|c|c|c|c|c|c|}
\hline Variable & $\begin{array}{l}1 \\
\text { Strongly } \\
\text { Disagree }\end{array}$ & Disagree & \begin{tabular}{l}
\multicolumn{1}{c}{3} \\
Neither Agree \\
Nor Disagree
\end{tabular} & Agree & $\begin{array}{c}5 \\
\text { Strongly } \\
\text { Agree }\end{array}$ \\
\hline Author1 & $8(11)$ & 12 (17) & $5(7)$ & $32(45)$ & $14(20)$ \\
\hline Author2 & $15(21)$ & $22(32)$ & $11(15)$ & 13 (18) & $10(14)$ \\
\hline Author3 & $9(13)$ & $15(21)$ & $6(8)$ & $25(35)$ & $16(23)$ \\
\hline Author4 & $5(7)$ & 27 (38) & $16(22)$ & $19(27)$ & $4(6)$ \\
\hline Author5 & $5(7)$ & $29(41)$ & $25(35)$ & 12 (17) & $0(0)$ \\
\hline \multicolumn{6}{|c|}{$\begin{array}{l}\text { Note. } \mathrm{N}=71 \text {. Author } 1=\mathrm{I} \text { am satisfied with my body; Author } 2=\mathrm{I} \text { am satisfied with the flatness of my } \\
\text { stomach, Author } 3=\mathrm{I} \text { am satisfied with the narrowness of my waist; Author } 4=\text { As long as my stomach is flat, } \\
\text { my weight does not matter; Author } 5=\text { as long as my waist is narrow, my weight does not matter. } \\
\text { Scores on Author } 1 \text { can range from } 1 \text { to } 5 \text {, with high scores indicating a higher level of body satisfaction. } \\
\text { Scores on Author } 2 \text { can range from } 1 \text { to } 5 \text {, with high scores indicating a higher level of satisfaction with } \\
\text { flatness of stomach. Scores on Author } 3 \text { can range from } 1 \text { to } 5 \text {, with high scores indicating a higher level of } \\
\text { satisfaction with narrowness of waist. Scores on Author } 4 \text { can range from } 1 \text { to } 5 \text {, with high scores } \\
\text { indicating a higher level of agreement with the idea that flatness of stomach is more important than weight. } \\
\text { Scores on Author } 5 \text { can range from } 1 \text { to 5, with high scores indicating a higher level of agreement with the } \\
\text { idea that narrowness of waist is more important than actual weight. }\end{array}$} \\
\hline
\end{tabular}




\section{Table 4}

Intercorrelations Between Variables: Items 28 and 29, Appearance

Evaluation subscale, Body Shape Questionnaire

Variable

$\mathrm{AE}$

BSQ
Item 28

Item 29

$.658^{* *}$

$-.751 * *$

$-.624 * *$
AVG

$.740 * *$

$-.780 * *$

Note. $\mathrm{N}=79$. Item 28, from the Body Areas Satisfaction subscale of the MBSRQ-AS measures satisfaction with mid torso lower torso (butt, hips, thighs, legs); Item 29 from the Body Areas Satisfaction subscale of the MBSRQ-AS, measures satisfaction with mid torso (waist and stomach) . AVG=the average of items 28 and 29. AE=Appearance Evaluation subscale of the MBSRQ-AS. BSQ=Body Shape Questionnaire. $* * \mathrm{p}<.01$ 


\section{Table 5}

Intercorrelations Between Variables: Author Constructed Items, Appearance Evaluation Subscale, Body Shape Questionnaire

Variable

$\mathrm{AE}$

BSQ
Author2

Author3

Note. $\mathrm{N}=79$ for AE and BSQ. $\mathrm{N}=71$ for Author2 and Author3. AE=Appearance Evaluation subscale of the MBSRQ-AS; Author2=I am satisfied with the flatness of my stomach, Author3=I am satisfied with the narrowness of my waist; BSQ=Body Shape Questionnaire. ${ }^{* *} \mathrm{p}<.01$ 


\section{Table 6}

Intercorrelations Between Variables: Items 28 and 29, Appearance Evaluation subscale, Discrepancy Score

Variable

Discrepancy
Item 28

$-.233$
Item 29

$-.390 *$
$\mathrm{AE}$

$-.358^{*}$

Note. $\mathrm{N}=65$ for Discrepancy. $\mathrm{N}=79$ for Item 28, Item 29, and AE. Discrepancy=discrepancy score calculated by subtracting ideal shape from actual on the Reese Figure Rating Scale; Item 28=item from the Body Areas Satisfaction subscale of the MBSRQ-AS that measures satisfaction with mid torso lower torso (butt, hips, thighs, legs); Item 29=item from the Body Areas Satisfaction subscale of the MBSRQ-AS that measures satisfaction with mid torso (waist and stomach); AE=Appearance Evaluation subscale of the MBSRQ-AS. $* \mathrm{p}<.05 ; * * \mathrm{p}<.01$. 


\section{Table 7}

Intercorrelations Between Variables: Author Constructed Items, Discrepancy Score

Variable Author2 Author3

Discrepancy $\quad-.488 * * \quad-.400 * *$

Note. $\mathrm{N}=65$ for Discrepancy. $\mathrm{N}=71$ for Author2 and Author 3. Discrepancy=discrepancy score calculated by subtracting ideal shape from actual on the Reese Figure Rating Scale; Author2=I am satisfied with the flatness of my stomach, Author $3=\mathrm{I}$ am satisfied with the narrowness of my waist. $* * \mathrm{p}<.01$. 
Rashanta A. Bledman was born July 29, 1983 in Los Angeles, CA. She graduated from Chapman University in 2005 with a Bachelor's Degree in Psychology and a Minor in Sociology. She earned a Master's degree in Counseling Psychology from the University of Missouri in 2007. She will complete her pre-doctoral internship at the University of Maryland Counseling Center in 2011, and plans to pursue a career in clinical practice. 